TRANSACTIONS OF THE

AMERICAN MATHEMATICAL SOCIETY

Volume 361, Number 4, April 2009, Pages 1703-1729

S 0002-9947(08)04458-9

Article electronically published on October 29, 2008

\title{
PARABOLIC KAZHDAN-LUSZTIG POLYNOMIALS FOR HERMITIAN SYMMETRIC PAIRS
}

\author{
FRANCESCO BRENTI
}

\begin{abstract}
We study the parabolic Kazhdan-Lusztig polynomials for Hermitian symmetric pairs. In particular, we show that these polynomials are always either zero or a monic power of $q$, and that they are combinatorial invariants.
\end{abstract}

\section{INTRODUCTION}

In their fundamental paper 13. Kazhdan and Lusztig defined, for any Coxeter group $W$, a family of polynomials, indexed by pairs of elements of $W$, which have become known as the Kazhdan-Lusztig polynomials of $W$ (see, e.g., 11, Chap. 7] or [2, Chap. 5]). These polynomials play an important role in several areas of mathematics, including the algebraic geometry and topology of Schubert varieties and representation theory (see, e.g., 2, Chap. 5], and the references cited there). In order to prove the existence of these polynomials Kazhdan and Lusztig introduced another family of polynomials, usually called the $R$-polynomials, whose interest lies mainly in the fact that their knowledge is equivalent to that of the Kazhdan-Lusztig polynomials.

In 1987 Deodhar ([7]) introduced parabolic analogues of all these polynomials. These parabolic Kazhdan-Lusztig and $R$-polynomials reduce to the ordinary ones for the trivial parabolic subgroup of $W$ and are also related to them in other ways (see, e.g., Proposition 2.4 below). Besides these connections the parabolic polynomials also play a direct role in several areas including the theories of generalized Verma modules ([6]), tilting modules ([15], [16]), quantized Schur algebras ([19]), Macdonald polynomials ([10, 9]), Schubert varieties in partial flag manifolds (12]), and in the representation theory of the Lie algebra $g l_{n}$ ([14).

The purpose of this work is to study the parabolic Kazhdan-Lusztig polynomials for Hermitian symmetric pairs. More precisely, we give explicit combinatorial product formulas for these polynomials which imply, in particular, that they are always either zero or a monic power of $q$, and that they are combinatorial invariants.

The organization of the paper is as follows. In the next section we recall definitions, notation and results that are used in the rest of this work. In $\S 3$ we introduce and study the main new combinatorial concept of this work, namely a class of (possibly skew) shifted partitions that we call shifted-Dyck. In $\S 4$, using the results in the previous section and some from [5], we prove the main results

Received by the editors August 7, 2006 and, in revised form, November 9, 2006.

2000 Mathematics Subject Classification. Primary 05E99; Secondary 20 F55.

The author was partially supported by EU grant HPRN-CT-2001-00272. Part of this research was carried out while the author was a member of the Mittag-Leffler Institut in Djürsholm, Sweden, whose hospitality and financial support are gratefully acknowledged.

(C)2008 American Mathematical Society 1703

Reverts to public domain 28 years from publication 
of this work. Namely, we give explicit combinatorial product formulas for the parabolic Kazhdan-Lusztig polynomials of Hermitian symmetric pairs. These imply that these polynomals are combinatorial invariants. Finally, in $\S 5$, we give some applications of our main results. More precisely, we derive combinatorial closed product formulas for certain alternating sums of ordinary Kazhdan-Lusztig polynomials, and we prove a combinatorial invariance result for the Kazhdan-Lusztig polynomials studied in 3 .

\section{Preliminaries}

In this section we collect some definitions, notation and results that are used in the rest of this work. We let $\mathbf{P} \stackrel{\text { def }}{=}\{1,2,3, \ldots\}$ and $\mathbf{N} \stackrel{\text { def }}{=} \mathbf{P} \cup\{0\}$. For $m, n \in \mathbf{N}$, $m \geq n$, we let $[n, m] \stackrel{\text { def }}{=}\{n, n+1, \ldots, m-1, m\}$ and $[n] \stackrel{\text { def }}{=}[1, n]($ where $[0] \stackrel{\text { def }}{=} \emptyset)$. The cardinality of a set $A$ will be denoted by $|A|$.

Given a set $T$ we let $S(T)$ be the set of all bijections $\pi: T \rightarrow T$, and $S_{n} \stackrel{\text { def }}{=} S([n])$. If $\sigma \in S_{n}$, then we write $\sigma$ in disjoint cycle form (see, e.g., [17, p. 17) and we usually omit writing the 1 -cycles of $\sigma$. So, for example, if $\sigma=(9,7,1,3,5)(2,6)$, then $\sigma(1)=3, \sigma(2)=6, \sigma(3)=5, \sigma(4)=4$, etc. Given $\sigma, \tau \in S_{n}$ we let $\sigma \tau \stackrel{\text { def }}{=} \sigma \circ \tau$ (composition of functions) so that, for example, $(1,2)(2,3)=(1,2,3)$.

We follow Chapter 3 of [17] for poset notation and terminology. In particular, given a poset $(P, \leq)$ and $u, v \in P$ we let $[u, v] \stackrel{\text { def }}{=}\{z \in P: u \leq z \leq v\}$ and call this an interval of $P$. We say that $v$ covers $u$, denoted $u \triangleleft v$ (or, equivalently, that $u$ is covered by $v)$ if $|[u, v]|=2$. The Hasse graph of $P$ is the graph having $P$ as vertex set and $\{\{u, v\} \subseteq P: u \triangleleft v$ or $v \triangleleft u\}$ as edge set. A poset is connected if its Hasse graph is a connected graph. The degree of an element $v \in P$ is its degree as a vertex of the Hasse graph, namely $\mid\{u \in P: u \triangleleft v$ or $v \triangleleft u\} \mid$. We say that $u, v \in P$ are comparable if either $u \leq v$ or $v \leq u$. If $P$ has a minimum element, denoted $\hat{0}$, then we call a subset of the form $[\hat{0}, u]$, for $u \in P$, a lower interval of $P$. We denote by $J(P)$ the set of order ideals of $P$, partially ordered by set inclusion. Given any $Q \subseteq P$ we will always consider $Q$ as a poset with the partial ordering induced by $P$ and call $Q$ a subposet of $P$. If $u, v \in P$ are such that $\{z \in P: z \geq u, z \geq v\}$ has a minimum element, then we call this minimum element the join of $u$ and $v$. Similarly, we define the meet of $u$ and $v$ if $\{z \in P: z \leq u, z \leq v\}$ has a maximum element. We say that $z \in P$ is minimal if there is no $v \in P, v \neq z$, such that $v \leq z$. Similarly, we define maximal. Given two posets $P$ and $Q$, we write $P \cong Q$ to mean that they are isomorphic as posets. We note the following elementary observation, whose verification is omitted.

Proposition 2.1. Let $P$ be a poset and $X, Y \in J(P), X \subseteq Y$. Then $[X, Y] \cong$ $J(Y \backslash X)$.

We follow $\S 7.2$ of [18] for any undefined notation and terminology concerning partitions. Let $\mathcal{H} \stackrel{\text { def }}{=}\left\{(i, j) \in \mathbf{P}^{2}: i \leq j\right\}$, with the ordering induced by the product ordering on $\mathbf{P}^{2}$. We call the finite order ideals of $\mathcal{H}$ shifted partitions. We denote by $\mathcal{S}$ the set of all finite order ideals of $\mathcal{H}$. We will always assume that $\mathcal{S}$ is partially ordered by set inclusion. It is well known that this makes $\mathcal{S}$ into a distributive lattice. Note that there is an obvious bijection between partitions into distinct parts and shifted partitions, given by

$$
\left(\lambda_{1}, \ldots, \lambda_{k}\right) \leftrightarrow\left\{(i, j) \in \mathbf{P}^{2}: 1 \leq i \leq k, i \leq j \leq \lambda_{i}+i-1\right\} .
$$


For this reason we will freely identify these objects, and use expressions such as "the shifted partition $(5,2,1)$." We also identify each shifted partition with its diagram, which we draw rotated counterclockwise by $\frac{3}{4} \pi$ radians with respect to the usual (Anglophone) convention. So, for example, the diagram of the shifted partition $(5,2,1)$ is shown in Figure 1 .

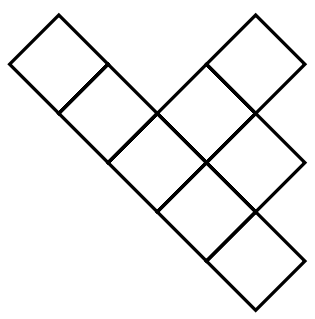

FiguRe 1

We call the elements of $\mathcal{H}$, and hence of a shifted partition, cells. Given a cell $x=(i, j) \in \mathcal{H}$ we define its level to be $l v(x) \stackrel{\text { def }}{=} i+j-1$. Expressions such as "leftmost" or "to the right of" always refer to the rotated diagrams. In particular, given a cell $x=(i, j) \in \mathcal{H}(i, j \geq 2)$ the cell directly below $x$ is the cell $x^{(1)} \stackrel{\text { def }}{=}(i-1, j-1)$ (see Figure 2). Similarly, we define directly above, directly northwest, etc... Also, we say that a cell $y=(k, l) \in \mathcal{H}$ is below $x$ if $l-k=j-i$ and $j \geq l$. We let $\delta_{n} \stackrel{\text { def }}{=}(n, n-1, \ldots, 2,1)$.

Let $\mu, \lambda \in \mathcal{S}, \mu \subseteq \lambda$. We then call $\lambda \backslash \mu$ a skew shifted partition. Note that, in poset theoretic language, skew shifted partitions are the finite convex subsets of $\mathcal{H}$. Given a skew shifted partition $\lambda \backslash \mu, \lambda=\left(\lambda_{1}, \lambda_{2}, \ldots\right)$ and $\mu=\left(\mu_{1}, \mu_{2}, \ldots\right)$, we define $l(\lambda \backslash \mu) \stackrel{\text { def }}{=}\left|\left\{i \in \mathbf{P}: \lambda_{i}>\mu_{i}\right\}\right|$. So, for example, $l((5,3,1) \backslash(4,3))=2$. Given a skew shifted partition $\eta$ and $x=(i, j) \in \mathcal{H}$ we define

$$
d_{\eta}(x) \stackrel{\text { def }}{=}|\{(k, l) \in \eta: l-k=j-i\}| .
$$

A connected skew shifted partition is uniquely determined, up to translation, by the two ordered sequences of the lengths of the sides of the "polygon" that it determines. For example, a skew shifted partition having the diagram depicted in Figure 2 is uniquely determined by the sequences $(4,2,1,2,2,1,1,1)$ and $(3,1,1,1,1,2,2,1,1,1)$ (in this order). We will use this "polygon notation" for skew shifted partitions in $\S 5$. Let $\eta$ be a connected skew shifted partition. We say that a cell $x=(i, j) \in \eta$ is an upper peak (respectively, lower valley) of $\eta$ if it is maximal (respectively, minimal) in $\eta$ and $i \neq j$. We call an element $x \in \eta$ an upper valley of $\eta$ if $x$ is covered by exactly two elements of $\eta$ whose join is not in $\eta$. Similarly, we define a lower peak. If $\eta$ and $\rho$ are two skew shifted partitions, then we write $\eta \approx \rho$ to mean that $\eta$ is a translate of $\rho$.

We say that a skew shifted partition is a border strip if it never contains both a cell and the cell directly above it. For brevity, we call a connected border strip a cbs. Let $\lambda, \mu \in \mathcal{S}$ be such that $\mu \subseteq \lambda$. The outer border strip $\theta$ of $\lambda \backslash \mu$ is the set of cells $x$ of $\lambda \backslash \mu$ such that the cell directly above $x$ is not in $\lambda \backslash \mu$. For example, the cells of the outer border strip of the skew shifted partition illustrated in Figure 2 are numbered from 1 to 13 . We will usually number the cells of $\theta$ consecutively 
from left to right in this way and identify them with their corresponding numbers. So, for example, 1 is the leftmost cell of $\theta$, and if $y \in \theta, y>1$, then $y-1$ is the cell of $\theta$ immediately to the left of $y$.

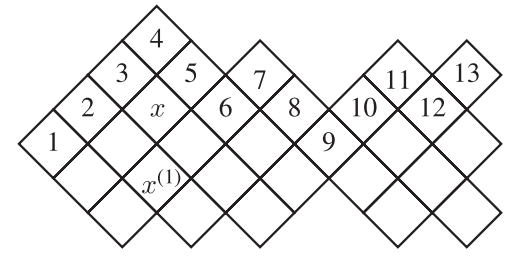

FIGURE 2

We follow [11] for general Coxeter groups notation and terminology. In particular, given a Coxeter system $(W, S)$ and $u \in W$ we denote by $l(u)$ the length of $u$ in $W$, with respect to $S$, and we let $D(u) \stackrel{\text { def }}{=}\{s \in S: l(u s)<l(u)\}$. For $u, v \in W$ we let $l(u, v) \stackrel{\text { def }}{=} l(v)-l(u)$. We denote by $e$ the identity of $W$, and we let $T \stackrel{\text { def }}{=}\left\{u s u^{-1}: u \in W, s \in S\right\}$ be the set of reflections of $W$. Given $J \subseteq S$ we let $W_{J}$ be the parabolic subgroup generated by $J$ and

$$
W^{J} \stackrel{\text { def }}{=}\{u \in W: l(s u)>l(u) \text { for all } s \in J\} .
$$

Note that $W^{\emptyset}=W$. If $W_{J}$ is finite, then we denote by $w_{0}(J)$ its longest element. We will always assume that $W^{J}$ is partially ordered by Bruhat order. Recall (see, e.g., [11, §5.9) that this means that $x \leq y$ if and only if there exist $r \in \mathbf{N}$ and $t_{1}, \ldots, t_{r} \in T$ such that $t_{r} \cdots t_{1} x=y$ and $l\left(t_{i} \cdots t_{1} x\right)>l\left(t_{i-1} \cdots t_{1} x\right)$ for $i=$ $1, \ldots, r$. Given $u, v \in W^{J}, u \leq v$, we let

$$
[u, v]^{J} \stackrel{\text { def }}{=}\left\{z \in W^{J}: u \leq z \leq v\right\}
$$

and $[u, v] \stackrel{\text { def }}{=}[u, v]^{\emptyset}$.

For $J \subseteq S, x \in\{-1, q\}$, and $u, v \in W^{J}$ we denote by $R_{u, v}^{J, x}(q)$ and $P_{u, v}^{J, x}(q)$ the parabolic R-polynomials and parabolic Kazhdan-Lusztig polynomials (respectively) of $W^{J}$ of type $x$ (we refer the reader to [7] for the definitions of these polynomials, see also Proposition 2.3 below). It follows immediately from [7, $\S \S 2-3]$ and from well-known facts (see, e.g., [11, $\S 7.5]$ and [11, $\S \S 7.9-11])$ that $R_{u, v}^{\emptyset,-1}(q)\left(=R_{u, v}^{\emptyset, q}(q)\right)$ and $P_{u, v}^{\emptyset,-1}(q)\left(=P_{u, v}^{\emptyset, q}(q)\right)$ are the (ordinary) R-polynomials and Kazhdan-Lusztig polynomials of $W$, which we will denote simply by $R_{u, v}(q)$ and $P_{u, v}(q)$, as customary.

The following result is due to Deodhar, and we refer the reader to [7 for its proof. For $u, v \in W^{J}$ let $\mu_{J, q}(u, v)$ be the coefficient of $q^{\frac{1}{2}(l(u, v)-1)}$ in $P_{u, v}^{J, q}(q)$ (so $\mu_{J, q}(u, v) \stackrel{\text { def }}{=} 0$ if $l(u, v)$ is even). We will often write $\mu(u, v)$ instead of $\mu_{J, q}(u, v)$ if there is no danger of confusion.

Proposition 2.2. Let $(W, S)$ be a Coxeter system, $J \subseteq S$, and $u, v \in W^{J}, u \leq v$. Then for each $s \in D(v)$ we have that

$$
P_{u, v}^{J, q}(q)=\tilde{P}-\sum_{\{u \leq w<v s: w s<w\}} \mu_{J, q}(w, v s) q^{\frac{l(w, v)}{2}} P_{u, w}^{J, q}(q),
$$


where

$$
\tilde{P}= \begin{cases}P_{u s, v s}^{J, q}+q P_{u, v s}^{J, q}, & \text { if } u s<u, \\ q P_{u s, v s}^{J, q}+P_{u, v s}^{J, q}, & \text { if } u<u s \in W^{J}, \\ 0, & \text { if } u<u s \notin W^{J} .\end{cases}
$$

The parabolic Kazhdan-Lusztig and $R$-polynomials are related to their ordinary counterparts in several ways, including the following one, which may be taken as their definition in most cases.

Proposition 2.3. Let $(W, S)$ be a Coxeter system, $J \subseteq S$, and $u, v \in W^{J}$. Then we have that

$$
R_{u, v}^{J, x}(q)=\sum_{w \in W_{J}}(-x)^{l(w)} R_{w u, v}(q),
$$

for all $x \in\{-1, q\}$, and

$$
P_{u, v}^{J, q}(q)=\sum_{w \in W_{J}}(-1)^{l(w)} P_{w u, v}(q) .
$$

Furthermore, if $W_{J}$ is finite, then

$$
P_{u, v}^{J,-1}(q)=P_{w_{0}(J) u, w_{0}(J) v}(q) .
$$

A proof of this result can be found in 7] (see Propositions 2.12 and 3.4, and Remark 3.8). Yet another relation (which, however, we will not use) between parabolic and ordinary Kazhdan-Lusztig polynomials is given in [7, Proposition $3.5]$.

The purpose of this work is to study the parabolic Kazhdan-Lusztig polynomials for quotients $W^{J}$ such that $\left(W, W_{J}\right)$ is a Hermitian symmetric pair. These quotients have been classified (see, e.g., [3]) and there are five infinite families and two exceptional ones. Using (and abusing slightly) the standard notation for the classification of the finite Coxeter systems, the Hermitian symmetric pairs are: $\left(A_{n}, A_{i-1} \times A_{n-i}\right),\left(B_{n}, A_{n-1}\right),\left(B_{n}, B_{n-1}\right),\left(D_{n}, A_{n-1}\right),\left(D_{n}, D_{n-1}\right),\left(E_{6}, D_{5}\right)$, $\left(E_{7}, E_{6}\right)(n \geq 3,1 \leq i \leq n)$. The parabolic Kazhdan-Lusztig polynomials for the pairs $\left(A_{n}, A_{i-1} \times A_{n-i}\right)(1 \leq i \leq n)$ have been computed in [4]. In this work we deal with the other pairs.

We follow [2, Chap. 8] for combinatorial descriptions of the Coxeter systems of types $B_{n}$ and $D_{n}$ as permutation groups. In particular, we let $S_{n}^{B}$ be the group of all bijections $w$ of $\{-n, \ldots,-1,1, \ldots, n\}$ to itself such that $w(-i)=-w(i)$ for all $i \in[n], s_{j} \stackrel{\text { def }}{=}(j, j+1)(-j,-j-1)$ for $j=1, \ldots, n-1, s_{0} \stackrel{\text { def }}{=}(1,-1)$, and $\mathcal{B}_{n} \stackrel{\text { def }}{=}\left\{s_{0}, \ldots, s_{n-1}\right\}$. If $v \in S_{n}^{B}$, then we write $v=\left[a_{1}, \ldots, a_{n}\right]$ to mean that $v(i)=a_{i}$, for $i=1, \ldots, n$. It is well known that $\left(S_{n}^{B}, \mathcal{B}_{n}\right)$ is a Coxeter system of type $B_{n}$ and that the following holds. Given $v \in S_{n}^{B}$ we let

$$
\begin{array}{r}
i n v(v) \stackrel{\text { def }}{=}\left|\left\{(i, j) \in[n]^{2}: i<j, v(i)>v(j)\right\}\right|, \\
N_{1}(v) \stackrel{\text { def }}{=}|\{i \in[n]: v(i)<0\}| \text { and } \\
N_{2}(v) \stackrel{\text { def }}{=}\left|\left\{(i, j) \in[n]^{2}: i<j, v(i)+v(j)<0\right\}\right| .
\end{array}
$$

Proposition 2.4. Let $v \in S_{n}^{B}$. Then $l(v)=i n v(v)+N_{1}(v)+N_{2}(v)$, and $D(v)=$ $\left\{s_{i} \in S_{B}: v(i)>v(i+1)\right\}$, where $v(0) \stackrel{\text { def }}{=} 0$. 
Let $\mathcal{A}_{n-1} \stackrel{\text { def }}{=}\left\{s_{1}, \ldots, s_{n-1}\right\}$. Then $\left(S_{n}^{B}\right)_{\mathcal{A}_{n-1}} \cong S_{n}$, and it is clear from Proposition 2.4 that $v \in\left(S_{n}^{B}\right)^{\mathcal{A}_{n-1}}$ if and only if $v^{-1}(1)<v^{-1}(2)<\cdots<v^{-1}(n)$. In this case,

$$
v^{-1}(1)<\cdots<v^{-1}(k)<0<v^{-1}(k+1)<\cdots<v^{-1}(n),
$$

where $k=N_{1}(v)$, and we associate to $v \in\left(S_{n}^{B}\right)^{\mathcal{A}_{n-1}}$ the shifted partition

$$
\Lambda_{B}(v) \stackrel{\text { def }}{=}\left(v^{-1}(-1), v^{-1}(-2), \ldots, v^{-1}(-k)\right) .
$$

The next three results are known, and not hard to prove.

Proposition 2.5. The map $\Lambda_{B}$ defined by (21) is a bijection between $\left(S_{n}^{B}\right)^{\mathcal{A}_{n-1}}$ and $\left\{\lambda \in \mathcal{S}: \lambda \subseteq \delta_{n}\right\}$. Furthermore $u \leq v$ in $\left(S_{n}^{B}\right)^{\mathcal{A}_{n-1}}$ if and only if $\Lambda_{B}(u) \subseteq \Lambda_{B}(v)$, and $l(v)=\left|\bar{\Lambda}_{B}(v)\right|$, for all $u, v \in\left(\bar{S}_{n}^{B}\right)^{\mathcal{A}_{n-1}}$.

We find it sometimes convenient to identify a shifted partition in $\{\lambda \in \mathcal{S}: \lambda \subseteq$ $\left.\delta_{n}\right\}$ with a lattice path with $(1,1)(u p)$ and $(1,-1)($ down $)$ steps starting at $(0,0)$ and having $n$ steps. So, for example, the shifted partition $(5,2,1) \subseteq \delta_{7}$ corresponds to the lattice path illustrated in Figure 3. Given such a lattice path $\lambda$ we denote by $\lambda_{-}$the lattice path which is the same as $\lambda$, except that its last (rightmost) step is down, and define $\lambda_{+}$similarly. Given $j \in[n-1]$ we say that a lattice path has a peak at $j$ if its $j$-th step (from the left) is up and its $(j+1)$-th step is down.

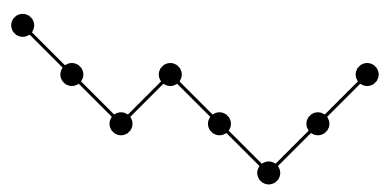

FiguRE 3

Proposition 2.6. Let $v \in\left(S_{n}^{B}\right)^{\mathcal{A}_{n-1}}$ and $i \in[n]$. Then the $i$-th step (from the left) of $\Lambda_{B}(v)$ (seen as a lattice path) is an up-step if and only if $v(n+1-i)<0$.

Lemma 2.7. Let $v \in\left(S_{n}^{B}\right)^{\mathcal{A}_{n-1}}$ and $j \in[n-1]$. Then $l\left(v s_{j}\right)<l(v)$ if and only if $\Lambda_{B}(v)$ has a peak at $n-j$. Furthermore, $l\left(v s_{0}\right)<l(v)$ if and only if the last step of $\Lambda_{B}(v)$ is up.

We let $S_{n}^{D}$ be the subgroup of $S_{n}^{B}$ defined by

$$
S_{n}^{D} \stackrel{\text { def }}{=}\left\{w \in S_{n}^{B}: N_{1}(w) \equiv 0 \quad(\bmod 2)\right\},
$$

$\tilde{s}_{0} \stackrel{\text { def }}{=}(1,-2)(2,-1)$, and $S_{D} \stackrel{\text { def }}{=}\left\{\tilde{s}_{0}, s_{1}, \ldots, s_{n-1}\right\}$. It is then well known that $\left(S_{n}^{D}, S_{D}\right)$ is a Coxeter system of type $D_{n}$, and that the following holds (see, e.g., [2, $\S 8.2])$.

Proposition 2.8. Let $v \in S_{n}^{D}$. Then $l(v)=i n v(v)+N_{2}(v)$, and $D(v)=\left\{s_{i} \in\right.$ $\left.S_{D}: v(i)>v(i+1)\right\}$, where $v(0) \stackrel{\text { def }}{=}-v(2)$.

Let $v \in S_{n}^{D}$. It is then clear from Proposition 2.8 that $v \in\left(S_{n}^{D}\right)^{\mathcal{A}_{n-1}}$ if and only if $v^{-1}(1)<\cdots<v^{-1}(n)$. In this case,

$$
v^{-1}(1)<\cdots<v^{-1}(k)<0<v^{-1}(k+1)<\cdots<v^{-1}(n),
$$


where $k=N_{1}(v)$, and we associate with $v \in\left(S_{n}^{D}\right)^{\mathcal{A}_{n-1}}$ the shifted partition

$$
\Lambda_{D}(v) \stackrel{\text { def }}{=}\left(v^{-1}(-1)-1, v^{-1}(-2)-1, \ldots, v^{-1}(-k)-1\right) .
$$

The next three results are known.

Proposition 2.9. The map $\Lambda_{D}$ defined by (44) is a bijection between $\left(S_{n}^{D}\right)^{\mathcal{A}_{n-1}}$ and $\left\{\lambda \in \mathcal{S}: \lambda \subseteq \delta_{n-1}\right\}$. Furthermore $u \leq v$ in $\left(S_{n}^{D}\right)^{\mathcal{A}_{n-1}}$ if and only if $\Lambda_{D}(u) \subseteq$ $\Lambda_{D}(v)$, and $l(v)=\left|\Lambda_{D}(v)\right|$, for all $u, v \in\left(S_{n}^{D}\right)^{\mathcal{A}_{n-1}}$.

Proposition 2.10. Let $v \in\left(S_{n}^{D}\right)^{\mathcal{A}_{n-1}}$ and $i \in[n-1]$. Then the $i$-th step (from the left) of $\Lambda_{D}(v)$ (seen as a lattice path) is an up-step if and only if $v(n+1-i)<0$. In particular, $v(1)<0$ if and only if $\Lambda_{D}(v)$ has an odd number of up-steps.

Lemma 2.11. Let $v \in\left(S_{n}^{D}\right)^{\mathcal{A}_{n-1}}$ and $j \in[2, n-1]$. Then $l\left(v s_{j}\right)<l(v)$ if and only if $\Lambda_{D}(v)$ has a peak at $n-j$. Furthermore, $l\left(v s_{1}\right)<l(v)$ (respectively, $l\left(v \tilde{s}_{0}\right)<l(v)$ ) if and only if the last step of $\Lambda_{D}(v)$ is up and $\Lambda_{D}(v)$ has an even (respectively, odd) number of up-steps.

Let $u \in S_{n}^{B}$, and $\mathcal{B}_{n-1} \stackrel{\text { def }}{=}\left\{s_{0}, \ldots, s_{n-2}\right\} \subseteq S_{B}$. Then $\left(S_{n}^{B}\right)_{\mathcal{B}_{n-1}} \cong S_{n-1}^{B}$ and by Proposition 2.4 we have that

$$
\left(S_{n}^{B}\right)^{\mathcal{B}_{n-1}}=\left\{w \in S_{n}^{B}: 0<w^{-1}(1)<\cdots<w^{-1}(n-1)\right\} .
$$

Therefore $u \in\left(S_{n}^{B}\right)^{\mathcal{B}_{n-1}}$ if and only if there exist $i \in[n]$ and $\varepsilon \in\{1,-1\}$ such that

$$
u=[1,2, \ldots, i-1, \varepsilon n, i, \ldots, n-1] .
$$

Let, for brevity, $u_{\varepsilon i} \stackrel{\text { def }}{=}[1,2, \ldots, i-1, \varepsilon n, i, \ldots, n-1]$. It then follows easily from the definition of Bruhat order that

$$
u_{n}<u_{n-1}<\cdots<u_{1}<u_{-1}<\cdots<u_{-n}
$$

So $\left(S_{n}^{B}\right)^{\mathcal{B}_{n-1}}$, partially ordered by Bruhat order, is a chain with $2 n$ elements.

Let $v \in S_{n}^{D}$, and $\mathcal{D}_{n-1} \stackrel{\text { def }}{=}\left\{\tilde{s}_{0}, s_{1}, \ldots, s_{n-2}\right\} \subseteq S_{D}$. Then $\left(S_{n}^{D}\right)_{\mathcal{D}_{n-1}} \cong S_{n-1}^{D}$ and by Proposition 2.8 we have that

$$
\left(S_{n}^{D}\right)^{\mathcal{D}_{n-1}}=\left\{w \in S_{n}^{D}: w^{-1}(-2)<w^{-1}(1)<w^{-1}(2)<\cdots<w^{-1}(n-1)\right\} .
$$

Hence, if $w \in\left(S_{n}^{D}\right)^{\mathcal{D}_{n-1}}$, then $0<w^{-1}(2)$. So

$$
\left(S_{n}^{D}\right)^{\mathcal{D}_{n-1}}=\left\{v_{\varepsilon i}: i \in[n], \varepsilon \in\{-1,1\}\right\},
$$

where

$$
v_{\varepsilon i} \stackrel{\text { def }}{=} \begin{cases}{[\varepsilon 1,2, \ldots, i-1, \varepsilon n, i, \ldots, n-1],} & \text { if } i \geq 2, \\ {[\varepsilon n, \varepsilon 1,2, \ldots, n-1],} & \text { if } i=1,\end{cases}
$$

for $i \in[n], \varepsilon \in\{-1,1\}$. Furthermore, it follows easily from the definition of Bruhat order (and from Proposition 2.8) that

$$
v_{n}<v_{n-1}<\cdots<v_{2}<v_{1}<v_{-2}<\cdots<v_{-n},
$$

$v_{2}<v_{-1}<v_{-2}$, and $v_{-1}$ and $v_{1}$ are incomparable. 


\section{Shifted Dyck Skew Shifted Partitions}

In this section we introduce and study the main new concept of this work, namely a class of skew shifted partitions that we call shifted Dyck. This class plays a fundamental role in the computation of the parabolic Kazhdan-Lusztig polynomials of Hermitian symmetric pairs, and it is the shifted analogue of the class of Dyck skew partitions introduced in 4. The results in this section are used in the next one in the proof of our main theorem.

Let $\theta \subseteq \mathcal{H}$ be a connected border strip (or, cbs, for short). Recall that we identify the cells of $\theta$ with the integers $1,2, \ldots,|\theta|$ as explained in $\S 2$. We say that $\theta$ is almost Dyck if $l v(x) \geq l v(1)$ for all $x \in \theta$. We say that $\theta$ is Dyck if it is almost Dyck and $l v(|\theta|)=l v(1)$.

Let $\theta$ be a cbs. Let $x$ be the rightmost cell of $\theta$ such that $l v(x)=l v(1)$. We define $\theta_{S} \stackrel{\text { def }}{=} \theta \cup\left\{y^{(1)}: x<y \leq|\theta|\right\}$ (recall that we denote by $y^{(1)}$ the cell directly below $y$ ). So, for example, if $\theta$ is the cbs depicted in Figure 4, then $\theta_{S}$ is depicted in Figure 5. Note that, if $\theta$ is almost Dyck, then $\theta=\theta_{S}$ if and only if $\theta$ is Dyck.

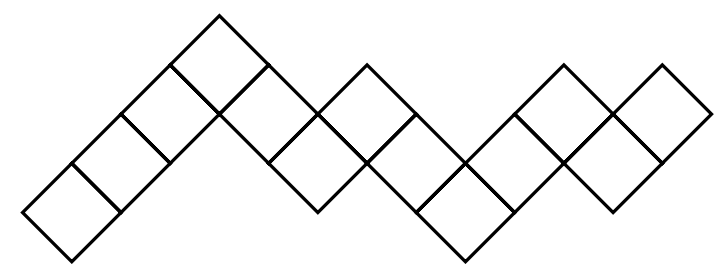

Figure 4

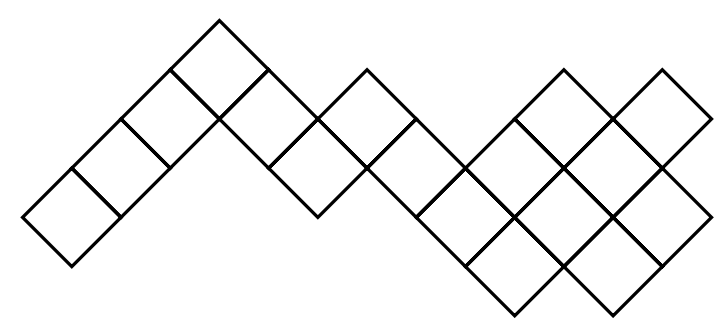

FigURE 5

We can now give the crucial definition of this work.

Let $\eta \subseteq \mathcal{H}$ be a skew shifted partition. We define $\eta$ to be shifted-Dyck (or, $s$-Dyck, for short) in the following inductive way:

i) $\eta$ is $s$-Dyck if and only if each one of its connected components is $s$-Dyck;

ii) if $\eta$ is connected, then $\eta$ is $s$-Dyck if and only if:

a) the outer border strip $\theta$ of $\eta$ is almost Dyck, $\theta_{S} \subseteq \eta$, and $\left|\theta_{S} \backslash \theta\right|$ is even;

b) $\eta \backslash \theta_{S}$ is $s$-Dyck.

Finally, $\emptyset$ is $s$-Dyck.

Let $\eta \subset \mathcal{H}$ be an $s$-Dyck skew shifted partition. We define the depth of $\eta$, denoted $d p(\eta)$, inductively by letting the depth of $\eta$ be the sum of the depths of 
its connected components, and if $\eta$ is connected, letting

$$
d p(\eta) \stackrel{\text { def }}{=} 1+d p\left(\eta \backslash \theta_{S}\right)
$$

(and $d p(\emptyset) \stackrel{\text { def }}{=} 0$ ), where $\theta$ is the outer border strip of $\eta$. So, for example, the skew shifted partition depicted in Figure 6 is not $s$-Dyck, while that depicted in Figure 2 is and has depth 6 .

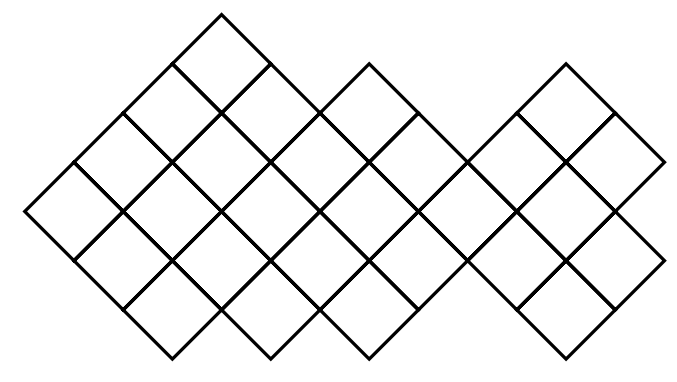

Figure 6

We begin with the following elementary property. Let $\eta \subseteq \mathcal{H}$ be a skew shifted partition with only one rightmost cell. We denote by $\eta^{\prime}$ any skew shifted partition whose diagram is the mirror image of that of $\eta$. Clearly, $\eta^{\prime}$ is defined only up to translation.

Proposition 3.1. Let $\eta$ be a skew shifted partition with only one rightmost cell. Then $\eta$ is $s$-Dyck if and only if $\eta^{\prime}$ is s-Dyck. Furthermore, in this case, $d p(\eta)=$ $d p\left(\eta^{\prime}\right)$.

Proof. Suppose $\eta$ is $s$-Dyck. We may clearly assume that $\eta$ is connected. We proceed by induction on $|\eta|$. Let $m$ be the unique rightmost cell of $\eta$. Then $m \in \theta$ (where $\theta$ is the outer border strip of $\eta$ ) while $m^{(1)} \notin \eta$ (by the uniqueness of $m$ ). Hence, since $\eta$ is $s$-Dyck, $l v(m)=l v(1)$. Therefore $\theta$ is Dyck, and $\eta \backslash \theta=\eta \backslash \theta_{S}$. But $\eta \backslash \theta_{S}$ is $s$-Dyck, and $\eta \backslash \theta_{S}$ has only one rightmost cell. Therefore, by our induction hypothesis, $\left(\eta \backslash \theta_{S}\right)^{\prime}$ is $s$-Dyck, and $d p\left(\left(\eta \backslash \theta_{S}\right)^{\prime}\right)=d p\left(\eta \backslash \theta_{S}\right)$. Hence $(\eta \backslash \theta)^{\prime}=\eta^{\prime} \backslash \theta^{\prime}$ is $s$-Dyck, and therefore $\eta^{\prime}$ is $s$-Dyck and $d p\left(\eta^{\prime}\right)=d p(\eta)$.

The next two technical lemmas are needed in the proof of the main result of this section (Theorem 3.5).

In what follows, given a skew shifted partition $\eta \subseteq \mathcal{H}$, we let, for brevity, $\eta^{(1)} \stackrel{\text { def }}{=}$ $\eta \backslash \theta_{S}$, where $\theta$ is the outer border strip of $\eta$.

Lemma 3.2. Let $\eta$ be a skew shifted partition that is $s$-Dyck, and $x$ be the leftmost cell of any connected component of $\eta^{(1)}$. Then

$$
l v(x) \geq l v(1)-d_{\theta_{S}}(x),
$$

where 1 is the leftmost cell of the connected component of $\eta$ containing $x$.

Proof. Suppose first that $d_{\theta_{S}}(x)=1$. Let $x^{\prime}$ be the cell directly above $x$ and $x^{\prime \prime}$ be the cell directly above $x^{\prime}$. Since $x^{\prime}, x \in \eta$, and $x$ is the leftmost cell of its connected component of $\eta^{(1)}$, we deduce that $y \in \eta \backslash \eta^{(1)}$, where $y$ is the cell directly northwest of $x$ (so, directly southwest of $\left.x^{\prime}\right)$. Hence $y \in \theta_{S}$ and hence $y \in \theta\left(\right.$ since $\left.d_{\theta_{S}}(x)=1\right)$. Therefore, since $\eta$ is $s$-Dyck, $l v(x)=l v(y)-1 \geq l v(1)-1$ and (5) holds. 
Suppose now that $d_{\theta_{S}}(x)=2$. Let $z$ be the rightmost cell of $\theta$ such that $\operatorname{lv}(z)=$ $\operatorname{lv}(1)$. Since $x, x^{\prime}, x^{\prime \prime} \in \eta$, by our hypothesis on $x$ we deduce similarly that $y \in \theta_{S} \backslash \theta$. Therefore $l v(x)=l v(y)-1 \geq l v(z)-2=l v(1)-2$, as desired.

Lemma 3.3. Let $\eta$ be a skew shifted partition that is $s$-Dyck, and $x \in \theta$. Then

$$
l v(x) \geq \operatorname{lv}(1)+d_{\eta}(x)-1,
$$

where 1 is the leftmost cell of the connected component of $\eta$ containing $x$.

Proof. We proceed by induction on $d_{\eta}(x)$, the result being clear if $d_{\eta}(x)=1$. Suppose $d_{\eta}(x) \geq 2$, and let

$$
\hat{x} \stackrel{\text { def }}{=} \begin{cases}x^{(1)}, & \text { if } d_{\theta_{S}}(x)=1, \\ x^{(2)}, & \text { if } d_{\theta_{S}}(x)=2,\end{cases}
$$

where $x^{(2)}$ is the cell directly below $x^{(1)}$. Then $\hat{x}$ is in the outer border strip of $\eta^{(1)}$ (unless $d_{\eta}(x)=d_{\theta_{S}}(x)=2$, in which case (6) is clear). Therefore, using our induction hypothesis, we obtain that

$$
\begin{aligned}
l v(x) & =\operatorname{lv}(\hat{x})+2 d_{\theta_{S}}(x) \\
& \geq \operatorname{lv}(\hat{1})+d_{\eta^{(1)}}(\hat{x})-1+2 d_{\theta_{S}}(x) \\
& =\operatorname{lv}(\hat{1})+d_{\eta}(x)-1+d_{\theta_{S}}(x) \\
& \geq \operatorname{lv}(1)+d_{\eta}(x)-1-d_{\theta_{S}}(\hat{1})+d_{\theta_{S}}(x)
\end{aligned}
$$

by Lemma 3.2 where $\hat{1}$ is the leftmost cell of the connected component of $\eta^{(1)}$ that contains $\hat{x}$, and (6) follows.

Proposition 3.4. Let $\eta$ be an s-Dyck skew shifted partition. Then below every upper peak of $\eta$ there is either a lower peak or a lower valley.

Proof. We proceed by induction on $|\eta|$. Let $x$ be an upper peak of $\eta$. Because $\eta$ is $s$-Dyck, $x$ cannot be the leftmost cell of $\eta$. Therefore $x-1, x+1 \in \eta$. If $x^{(1)} \notin \eta$, then clearly we are done. So assume $x^{(1)} \in \eta$. Let $\theta$ be the (almost Dyck, by our hypothesis) outer border strip of $\eta$. If $d_{\theta_{S}}(x)=1$, then $x^{(1)}$ is an upper peak of $\eta \backslash \theta_{S}$ and hence, by induction, we are done. So assume $d_{\theta_{S}}(x)=2$. Then $x^{(1)} \in \theta_{S}$ and, since $\eta$ is $s$-Dyck, $d_{\eta}(x+1) \geq 2$. If $d_{\eta}(x-1)=1$, then $d_{\theta_{S}}(x-1)=1$, so, since $\eta$ is $s$-Dyck, $x-1$ would be the rightmost cell of $\theta$ such that $l v(x-1)=l v(1)$, which is a contradiction since $l v(x+1)=l v(x-1)$. So $d_{\eta}(x-1) \geq 2$. Let $x^{(2)}$ be the cell directly below $x^{(1)}$. If $x^{(2)} \notin \eta$, then clearly we are done, else $x^{(2)}$ is an upper peak of $\eta \backslash \theta_{S}$ and the result again follows by induction.

We can now prove the main result of this section.

Theorem 3.5. Let $\eta$ be a skew shifted partition, and $x$ be an upper peak of $\eta$. Suppose that $\eta$ has a lower valley, $y$, below $x, y \neq x$. Then the following are equivalent:

i) $\eta$ is $s$-Dyck;

ii) exactly one of $\eta \backslash\{x\}, \eta \backslash\{x, y\}$ is $s$-Dyck;

iii) $\eta \backslash\{y\}$ is s-Dyck.

Furthermore, in this case,

$$
d p(\eta \backslash\{y\})+1=d p(\eta)= \begin{cases}d p(\eta \backslash\{x\})+1, & \text { if } \eta \backslash\{x\} \text { is s-Dyck, } \\ d p(\eta \backslash\{x, y\}), & \text { if } \eta \backslash\{x, y\} \text { is s-Dyck. }\end{cases}
$$


Proof. We may clearly assume that $\eta$ is connected. Note that $\eta$ and $\eta \backslash\{y\}$ have the same outer border strip, which we denote by $\theta$. Let $z$ be the rightmost cell of $\theta$ such that $l v(z)=l v(1)$.

We prove the equivalence of i) and iii) first.

We proceed by induction on $d_{\eta}(x)$. Suppose $d_{\eta}(x)=2$. If $z \leq x$, then, since $d_{\eta}(x+1)=1, \theta_{S} \nsubseteq \nsubseteq \eta$ and $\theta_{S} \nsubseteq \nsubseteq \eta \backslash\{y\}$ and the result holds. If $z \geq x+1$, then $\theta_{S} \subseteq \eta$ if and only if $\theta_{S} \subseteq \eta \backslash\{y\}$ and the single cell $y$ is a connected component of $\eta^{(1)}$ but not of $(\eta \backslash\{y\})^{(1)}$, which have the same connected components otherwise. So $\eta^{(1)}$ is $s$-Dyck if and only if $(\eta \backslash\{y\})^{(1)}$ is $s$-Dyck, and in this case, $d p\left(\eta^{(1)}\right)=d p\left((\eta \backslash\{y\})^{(1)}\right)+1$, and the result follows.

Suppose now that $d_{\eta}(x) \geq 3$.

If $z \geq x+1$, then $x^{(1)}$ is an upper peak of $\eta^{(1)}$, and $\eta^{(1)}$ has the lower valley $y$ below $x^{(1)}$. Therefore, by the induction hypothesis, $\eta^{(1)}$ is $s$-Dyck if and only if $\eta^{(1)} \backslash\{y\}$ is, and the result follows since $\eta^{(1)} \backslash\{y\}=(\eta \backslash\{y\})^{(1)}$.

If $z=x$, then $\theta$ is not almost Dyck, so the result holds.

If $z \leq x-1$, then $z<x-1$ and therefore $x^{(2)}$ (cell directly below $x^{(1)}$ ) is an upper peak of $\eta^{(1)}$, and $\eta^{(1)}$ has the lower valley $y$ below $x^{(2)}$. The result hence follows by induction as in the previous case if $x^{(2)} \neq y$, and directly if $x^{(2)}=y$.

We now prove the equivalence of i) and ii).

Note first that, since $y \neq x, x>1$. If $\operatorname{lv}(1) \geq \operatorname{lv}(x-1)-d_{\eta}(x)+3$, then, since $x-1$ is in the outer border strips of all of $\eta, \eta \backslash\{x\}$, and $\eta \backslash\{x, y\}$, and $d_{\eta}(x-1)=d_{\eta \backslash\{x\}}(x-1)=d_{\eta \backslash\{x, y\}}(x-1)=d_{\eta}(x)-1$, we conclude from Lemma 3.3 that none of $\eta, \eta \backslash\{x\}$, and $\eta \backslash\{x, y\}$ are $s$-Dyck and the result holds.

We may therefore assume that

$$
l v(1) \leq l v(x-1)-d_{\eta}(x)+2 .
$$

We now proceed by induction on $d_{\eta}(x)$. We denote by $\theta^{\prime}$ (respectively, $\theta^{\prime \prime}$ ) the outer border strip of $\eta \backslash\{x\}$ (respectively, $\eta \backslash\{x, y\}$ ).

If $d_{\eta}(x)=2$, then $l v(1) \leq l v(x-1)$. If $l v(1)=l v(x-1)$, then $\eta \backslash\{x\}$ is not $s$-Dyck (since $\theta^{\prime}$ is not almost Dyck), and it is easy to verify that $\eta$ is $s$-Dyck if and only if $\eta \backslash\{x, y\}$ is, and in this case, $d p(\eta)=d p(\eta \backslash\{x, y\})$, so the result holds in this case.

If $l v(1)<l v(x-1)$, then $\eta \backslash\{x, y\}$ is not $s$-Dyck (since $\left.(x-1)^{(1)} \notin \eta \backslash\{x, y\}\right)$. If $z<x-1$, then $\eta$ and $\eta \backslash\{x\}$ are not $s$-Dyck (since $d_{\eta}(x-1)=d_{\eta \backslash\{x\}}(x-1)=1$ ) and the result holds. So assume $z>x+1$. Then $\theta^{\prime}=(\theta \backslash\{x\}) \cup\{y\}$. Hence $\theta^{\prime}$ is almost Dyck if and only if $\theta$ is. Also, since $z>x+1, \theta_{S}^{\prime}=\left(\theta_{S} \backslash\{x\}\right) \cup\{y\}$ so $\theta_{S} \subseteq \eta$ if and only if $\theta_{S}^{\prime} \subseteq \eta \backslash\{x\}$ and $\left|\theta_{S} \backslash \theta\right|=\left|\theta_{S}^{\prime} \backslash \theta^{\prime}\right|$. Furthermore $\eta^{(1)}$ is $s$-Dyck if and only if $(\eta \backslash\{x\})^{(1)}$ is $s$-Dyck (since they are equal except for one connected component equal to $\{y\}$ ), so we conclude that $\eta$ is $s$-Dyck if and only if $\eta \backslash\{x\}$ is, and in this case $d p(\eta)=d p(\eta \backslash\{x\})+1$, and the result holds.

Assume now that $d_{\eta}(x)=3$. Then

$$
l v(1) \leq l v(x-1)-1 .
$$

If $l v(1)=l v(x-1)-1$, then by Lemma 3.3, applied to $x^{(1)}, \eta \backslash\{x\}$ is not $s$-Dyck.

If $z<x-1$, then (since $l v(x)>l v(1)$, see Figure 7) $l v(w)>l v(1)$ for all $w \in \theta$, $w>z$. Also,

$$
\theta_{S}^{\prime \prime}=(\theta \backslash\{x\}) \cup\left\{x^{(1)}\right\} \cup\left\{w^{(1)}: w \in \theta, w>x\right\} .
$$




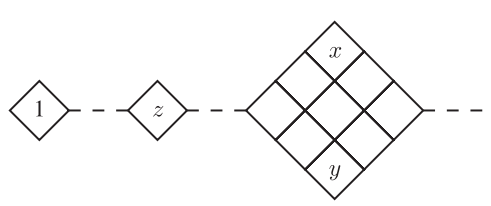

Figure 7

Suppose $\eta$ is $s$-Dyck. Then $\theta$ is almost Dyck, $\theta_{S} \subseteq \eta,\left|\theta_{S} \backslash \theta\right|$ is even, and $\eta \backslash \theta_{S}$ is $s$ Dyck. Therefore, $\theta^{\prime \prime}=(\theta \backslash\{x\}) \cup\left\{x^{(1)}\right\}$ is almost Dyck and, by (8), $\theta_{S}^{\prime \prime} \subseteq \eta \backslash\{x, y\}$ and $\left|\theta_{S}^{\prime \prime} \backslash \theta^{\prime \prime}\right|=|\theta|-x \equiv|\theta|-z=\left|\theta_{S} \backslash \theta\right|(\bmod 2)$ (since $\left.l v(z)=l v(x)-2\right)$. Furthermore, $\eta^{(1)}$ and $(\eta \backslash\{x, y\})^{(1)}$ have the same connected components except for the connected component $\eta_{0}$ of $(\eta \backslash\{x, y\})^{(1)}$ that contains $(z+1)^{(1)}$ (note that $(z+1)^{(1)} \in \eta$ because $(z+1)^{(1)} \in \theta_{S}$, but $z^{(1)} \notin \eta$, by Lemma 3.3. since $\eta$ is $s$-Dyck), which is replaced by $\eta_{0} \backslash\left\{w^{(1)}, w \in \theta, z<w<x\right\}$ and $\{y\}$ in $\eta^{(1)}$. But $\eta_{0} \backslash\left\{w^{(1)}: w \in \theta, z<w<x\right\}$ is $s$-Dyck and $\left\{w^{(1)}: w \in \theta, z<w<x\right\}$ is a Dyck $c b s$ and is the outer border strip of $\eta_{0}$. Therefore $\eta_{0}$ is $s$-Dyck and hence $(\eta \backslash\{x, y\})^{(1)}$ is $s$-Dyck, so $\eta \backslash\{x, y\}$ is $s$-Dyck and in this case, $d p(\eta)=d p(\eta \backslash\{x, y\})$, as desired.

Conversely, suppose $\eta \backslash\{x, y\}$ is $s$-Dyck. Then $\theta^{\prime \prime}$ is almost Dyck, $\theta_{S}^{\prime \prime} \subseteq \eta \backslash\{x, y\}$, $\left|\theta_{S}^{\prime \prime} \backslash \theta^{\prime \prime}\right|$ is even, and $(\eta \backslash\{x, y\})^{(1)}$ is $s$-Dyck. Therefore $\theta$ is almost Dyck, and $\eta_{0}$ is $s$-Dyck (where $\eta_{0}$ is the connected component of $(\eta \backslash\{x, y\})^{(1)}$ containing $\left.(x-1)^{(1)}\right)$. Since $\eta_{0}$ is $s$-Dyck, its outer border strip, $\theta_{0}$, is almost Dyck; therefore the leftmost cell $a^{(1)}$ of $\theta_{0}$ must satisfy $l v\left(a^{(1)}\right) \leq l v\left((x-1)^{(1)}\right)(=l v(z)-1)$. On the other hand, $a$ (the cell directly above $a^{(1)}$ ) is in $\theta$, and $z<a<x$, so $l v\left(a^{(1)}\right)=l v(a)-2 \geq l v(z)-1$. Therefore $l v\left(a^{(1)}\right)=l v(z)-1$ and hence $\theta_{0}$ is Dyck, and $a=z+1$. Therefore $\theta_{0}=\left\{w^{(1)}: w \in \theta, z<w<x\right\}$. Since $\theta_{0}$ is a Dyck $c b s$ we conclude that $\eta_{0} \backslash \theta_{0}$ is $s$-Dyck. Therefore $\theta_{S} \subseteq \eta$, and $\left|\theta_{S} \backslash \theta\right|=|\theta|-z \equiv|\theta|-x=\left|\theta_{S}^{\prime \prime} \backslash \theta^{\prime \prime}\right| \equiv 0$ $(\bmod 2)$. Furthermore, $\eta^{(1)}$ and $(\eta \backslash\{x, y\})^{(1)}$ are equal except for $\eta_{0}$, which is replaced by $\eta_{0} \backslash \theta_{0}$ and $\{y\}$ in $\eta^{(1)}$. Hence $\eta^{(1)}$ is $s$-Dyck and therefore $\eta$ is $s$-Dyck, and $d p\left(\eta_{0}\right)=d p\left(\eta_{0} \backslash \theta_{0}\right)+1=d p\left(\left(\eta_{0} \backslash \theta_{0}\right) \cup\{y\}\right)$, so $d p(\eta)=d p(\eta \backslash\{x, y\})$, as desired.

If $z>x+1$, then

$$
\theta_{S}=\theta \cup\left\{w^{(1)}: w \in \theta, z<w\right\}
$$

while

$$
\theta_{S}^{\prime \prime}=\left(\theta_{S} \backslash\{x\}\right) \cup\left\{x^{(1)}\right\} .
$$

This implies that $\theta$ is almost Dyck if and only if $\theta^{\prime \prime}$ is (and we may assume that this is the case), that $\left|\theta_{S}^{\prime \prime} \backslash \theta^{\prime \prime}\right|=\left|\theta_{S} \backslash \theta\right|$ and that $\theta_{S} \subseteq \eta$ if and only if $\theta_{S}^{\prime \prime} \subseteq \eta \backslash\{x, y\}$. Furthermore,

$$
(\eta \backslash\{x, y\})^{(1)}=\eta^{(1)} \backslash\left\{x^{(1)}, y\right\} .
$$

So we only have to show that $\eta^{(1)}$ is $s$-Dyck if and only if $\eta^{(1)} \backslash\left\{x^{(1)}, y\right\}$ is. But $\eta^{(1)}$ is a skew shifted partition, and $x^{(1)}$ is an upper peak of $\eta^{(1)}$ that has the lower valley $y$ below it. Let $a^{(1)}$ be the leftmost cell of the connected component of $\eta^{(1)}$ that contains $y$. Then $a$ (the cell directly above $\left.a^{(1)}\right)$ is in $\theta$ and $l v(a-1)<l v(a)$ by the choice of $a^{(1)}$. But $a-1 \in \theta$; hence $l v(a-1) \geq l v(1)$ (we are assuming that $\theta$ is almost Dyck) and therefore $l v(a)>l v(1)$, which implies that $l v\left(a^{(1)}\right) \geq$ 
$l v(1)-1=l v\left((x-1)^{(1)}\right)>l(y)$. Therefore, $\eta^{(1)} \backslash\left\{x^{(1)}\right\}$ is not $s$-Dyck. Hence, since $d_{\eta^{(1)}}\left(x^{(1)}\right)=2$, by what has been proved above, we conclude that $\eta^{(1)}$ is $s$-Dyck if and only if $\eta^{(1)} \backslash\left\{x^{(1)}, y\right\}$ is, and that $d p\left(\eta^{(1)}\right)=d p\left(\eta^{(1)} \backslash\left\{x^{(1)}, y\right\}\right)$, so (7) follows from (11).

Suppose now that $l v(1) \leq l v(x-1)-2$. If $z<x-1$, then

$$
\theta^{\prime \prime}=\theta^{\prime}=(\theta \backslash\{x\}) \cup\left\{x^{(1)}\right\}
$$

and

$$
\theta_{S}^{\prime \prime}=\theta_{S}^{\prime}=\left(\theta_{S} \backslash\{x\}\right) \cup\{y\} .
$$

Therefore $\theta_{S}^{\prime \prime} \nsubseteq \eta \backslash\{x, y\}$ and hence $\eta \backslash\{x, y\}$ is not $s$-Dyck. Furthermore, note that $\theta$ is almost Dyck if and only if $\theta^{\prime}$ is, $\left|\theta_{S} \backslash \theta\right|=\left|\theta_{S}^{\prime} \backslash \theta^{\prime}\right|$, and $\theta_{S} \subseteq \eta$ if and only if $\theta_{S}^{\prime} \subseteq \eta \backslash\{x\}$. Finally, $\eta^{(1)}$ and $(\eta \backslash\{x\})^{(1)}$ are equal except for the connected component $\{y\}$ that appears in $\eta^{(1)}$ but not in $(\eta \backslash\{x\})^{(1)}$, so we conclude that $\eta$ is $s$ Dyck if and only if $\eta \backslash\{x\}$ is $s$-Dyck, and $d p(\eta)=d p\left(\eta^{(1)}\right)+1=d p\left((\eta \backslash\{x\})^{(1)}\right)+2=$ $d p(\eta \backslash\{x\})+1$, and the result holds.

If $z>x+1$, then

$$
\theta^{\prime \prime}=\theta^{\prime}=(\theta \backslash\{x\}) \cup\left\{x^{(1)}\right\}
$$

and

$$
\theta_{S}^{\prime \prime}=\theta_{S}^{\prime}=\left(\theta_{S} \backslash\{x\}\right) \cup\left\{x^{(1)}\right\} .
$$

Therefore $\theta$ is almost Dyck if and only if $\theta^{\prime}$ is, $\theta_{S} \backslash \theta=\theta_{S}^{\prime \prime} \backslash \theta^{\prime \prime}=\theta_{S}^{\prime} \backslash \theta^{\prime}$, and $\theta_{S} \subseteq \eta$ if and only if $\theta_{S}^{\prime} \subseteq \eta \backslash\{x\}$ if and only if $\theta_{S}^{\prime \prime} \subseteq \eta \backslash\{x, y\}$. Furthermore,

$$
(\eta \backslash\{x\})^{(1)}=\eta^{(1)} \backslash\left\{x^{(1)}\right\}
$$

and

$$
(\eta \backslash\{x, y\})^{(1)}=\eta^{(1)} \backslash\left\{x^{(1)}, y\right\} .
$$

But $x^{(1)}$ is an upper peak of $\eta^{(1)}$ that has the lower valley, $y$, below it, and $d_{\eta^{(1)}}\left(x^{(1)}\right)=2$, so by our induction hypothesis we conclude that $\eta^{(1)}$ is $s$-Dyck if and only if either $\eta^{(1)} \backslash\left\{x^{(1)}\right\}$ or $\eta^{(1)} \backslash\left\{x^{(1)}, y\right\}$ are $s$-Dyck, but not both, and that in this case either $d p\left(\eta^{(1)} \backslash\left\{x^{(1)}, y\right\}\right)=d p\left(\eta^{(1)}\right)$ (if $\eta^{(1)} \backslash\left\{x^{(1)}, y\right\}$ is $s$-Dyck) or $d p\left(\eta^{(1)} \backslash\left\{x^{(1)}\right\}\right)=d p\left(\eta^{(1)}\right)-1$ (if $\eta^{(1)} \backslash\left\{x^{(1)}\right\}$ is $s$-Dyck) and the result follows from (12) and (13).

Suppose now that $d_{\eta}(x) \geq 4$. Then

$$
l v(1) \leq l v(x-1)-2 .
$$

Hence $\theta^{\prime \prime}=\theta^{\prime}=(\theta \backslash\{x\}) \cup\left\{x^{(1)}\right\}$. If $\theta$ is not almost Dyck, then the result follows immediately. So assume that $\theta$ is almost Dyck. Let, for convenience,

$$
\hat{x} \stackrel{\text { def }}{=} \begin{cases}x^{(1)}, & \text { if } z>x+1, \\ x^{(2)}, & \text { if } z<x-1,\end{cases}
$$

where $x^{(2)}$ is the cell directly below $x^{(1)}$. Then

$$
\theta_{S}^{\prime \prime}=\theta_{S}^{\prime}=\left(\theta_{S} \backslash\{x\}\right) \cup\{\hat{x}\} .
$$

It then follows from (14) that

$$
(\eta \backslash\{x\})^{(1)}=\eta^{(1)} \backslash\{\hat{x}\}
$$

and

$$
(\eta \backslash\{x, y\})^{(1)}=\eta^{(1)} \backslash\{\hat{x}, y\} .
$$


Now, from (14) we have that $\theta_{S} \subseteq \eta$ if and only if $\theta_{S}^{\prime} \subseteq \eta \backslash\{x\}$ if and only if $\theta_{S}^{\prime \prime} \subseteq \eta \backslash\{x, y\}$, and $\left|\theta_{S} \backslash \theta\right|=\left|\theta_{S}^{\prime} \backslash \theta^{\prime}\right|=\left|\theta_{S}^{\prime \prime} \backslash \theta^{\prime \prime}\right|$. Furthermore, $\theta^{\prime}=\theta^{\prime \prime}$ is almost Dyck. Finally, observe that $\hat{x}$ is an upper peak of $\eta^{(1)}$ that has the lower valley $y$ below it. Hence, by the induction hypothesis, $\eta^{(1)}$ is $s$-Dyck if and only if either $\eta^{(1)} \backslash\{\hat{x}\}$ or $\eta^{(1)} \backslash\{\hat{x}, y\}$ are $s$-Dyck, but not both, and in this case either $d p\left(\eta^{(1)}\right)=d p\left(\eta^{(1)} \backslash\{\hat{x}, y\}\right)$ (if $\eta^{(1)} \backslash\{\hat{x}, y\}$ is $s$-Dyck) or $d p\left(\eta^{(1)}\right)=d p\left(\eta^{(1)} \backslash\{\hat{x}\}\right)+1$ (if $\eta^{(1)} \backslash\{\hat{x}\}$ is $s$-Dyck), so the result follows from (15) and (16).

We note the following consequence of the proof of the previous theorem, which will be used in the sequel.

Proposition 3.6. Let $\eta$ be a skew shifted partition, and $x$ be an upper peak of $\eta$. Suppose that $\eta$ has a lower valley, $y$, below $x, y \neq x$. Then $\eta \backslash\{x\}$ and $\eta \backslash\{x, y\}$ cannot both be s-Dyck.

We conclude this section with three technical lemmas which are needed in $\S 5$.

Lemma 3.7. Let $n \in \mathbf{P}, n \geq 2$, and $\mu$ be a shifted partition such that $\mu \subseteq \delta_{n}$. Then $\delta_{n} \backslash \mu$ is $s$-Dyck if and only if there exist $0 \leq i_{1}<\cdots<i_{r} \leq\left\lfloor\frac{n-1}{2}\right\rfloor\left(0 \leq r \leq\left\lfloor\frac{n+1}{2}\right\rfloor\right)$ such that

$$
\mu= \begin{cases}\left(2 i_{r}+1,2 i_{r}, \ldots, 2 i_{1}+1,2 i_{1}\right), & \text { if } n \text { is odd }, \\ \left(n, 2 i_{r}+1,2 i_{r}, \ldots, 2 i_{1}+1,2 i_{1}\right), & \text { if } n \text { is even. }\end{cases}
$$

In this case, $d p\left(\delta_{n} \backslash \mu\right)=\left\lceil\frac{n-l(\mu)}{2}\right\rceil$.

Proof. We proceed by induction on $n$. If $n \leq 3$, the result is easy to verify. Let $\mu=\left(\mu_{1}, \mu_{2}, \ldots, \mu_{l}\right)$.

Suppose that $\delta_{n} \backslash \mu$ is $s$-Dyck. If $\mu_{1}=n$, then $\delta_{n} \backslash \mu \approx \delta_{n-1} \backslash\left(\mu_{2}, \ldots, \mu_{l}\right)$ and by induction the result holds. If $\mu_{1}<n$, then the outer border strip $\theta$ of $\delta_{n} \backslash \mu$ is a $1 \times n$ rectangle such that $l v(i)=l v(1)+i-1$ for $i=1, \ldots, n$. Therefore, since $\delta_{n} \backslash \mu$ is $s$-Dyck, we deduce that $\mu_{1} \leq n-2$ and $n$ is odd. Hence $\mu \subseteq \delta_{n-2}$, and $\delta_{n-2} \backslash \mu=\left(\delta_{n} \backslash \mu\right) \backslash \theta_{S}$, so the result follows by induction.

Conversely, suppose $\mu$ is of the form (17). If $\mu_{1}=n$, then $\delta_{n} \backslash \mu \approx \delta_{n-1} \backslash$ $\left(\mu_{2}, \ldots, \mu_{l}\right)$ and we are done by induction. If $\mu_{1}<n$, then $n$ is odd so, by (17), $\mu \subseteq \delta_{n-2}$. Hence $\theta$ (the outer border strip of $\delta_{n} \backslash \mu$ ) is almost Dyck, $\theta_{S} \subseteq \delta_{n} \backslash \mu$ and $\left|\theta_{S} \backslash \theta\right|=n-1$ is even. Furthermore, $\left(\delta_{n} \backslash \mu\right) \backslash \theta_{S}=\delta_{n-2} \backslash \mu$ and this is $s$-Dyck by induction. Hence $\delta_{n} \backslash \mu$ is $s$-Dyck, as desired.

In what follows we will sometimes call, for brevity, a shifted partition $\mu$ satisfying the equivalent conditions of Lemma 3.7 almost even.

Lemma 3.8. Let $n \in \mathbf{P}, n \geq 3, \nu_{n} \stackrel{\text { def }}{=}(n, n-1, \ldots, 4,3,1)$, and $\mu$ be a shifted partition such that $\mu \subseteq \nu_{n}$. Then $\nu_{n} \backslash \mu$ is s-Dyck if and only if there exist $2 \leq i_{1}<$ $\cdots<i_{r} \leq\left\lfloor\frac{n-1}{2}\right\rfloor\left(0 \leq r \leq\left\lfloor\frac{n-3}{2}\right\rfloor\right)$ and $(a, b) \in\{(3,1),(2,1),(2,0),(3,0)\}$ such that

$$
\mu= \begin{cases}\left(2 i_{r}+1,2 i_{r}, \ldots, 2 i_{1}+1,2 i_{1}, a, b\right), & \text { if } n \text { is odd } \\ \left(n, 2 i_{r}+1,2 i_{r}, \ldots, 2 i_{1}+1,2 i_{1}, a, b\right), & \text { if } n \text { is even. }\end{cases}
$$

In this case, $d p\left(\nu_{n} \backslash \mu\right)=\left\lceil\frac{n-l(\mu)}{2}\right\rceil+d p((3,1) \backslash(a, b))-1$.

Proof. We proceed by induction on $n$. If $n \leq 4$ the result is easy to verify. Let $\mu=\left(\mu_{1}, \mu_{2}, \ldots, \mu_{l}\right)$. 
Suppose that $\nu_{n} \backslash \mu$ is $s$-Dyck. If $\mu_{1}=n$, then $\nu_{n} \backslash \mu \approx \nu_{n-1} \backslash\left(\mu_{2}, \ldots, \mu_{l}\right)$ and by induction the result holds. If $\mu_{1}<n$, then, since $\nu_{n} \backslash \mu$ is $s$-Dyck, $\mu_{1} \leq n-2$ so the outer border strip $\theta$ of $\nu_{n} \backslash \mu$ is a cbs of cardinality $n$ such that $l v(i)=l v(1)+i-1$ for $i=1, \ldots, n-2, l v(n-1)=l v(n-2)-1, l v(n)=l v(n-2)$. Therefore, since $\nu_{n} \backslash \mu$ is $s$-Dyck, we deduce that $\mu_{n-1}=0, \mu_{n-2}=0, \mu_{n-3} \leq 1$, and $n$ is odd. Hence $\mu \subseteq \nu_{n-2}$ and $\nu_{n-2} \backslash \mu=\left(\nu_{n} \backslash \mu\right) \backslash \theta_{S}$, so the result follows by induction.

Conversely, suppose $\mu$ is of the form (18). If $\mu_{1}=n$, then $\nu_{n} \backslash \mu \approx \nu_{n-1} \backslash$ $\left(\mu_{2}, \ldots, \mu_{n-1}\right)$ and we are done by induction. If $\mu_{1}<n$, then $n$ is odd, so, by (18), $\mu_{1} \leq n-2$ and hence $\mu_{n-4} \leq 3$. This, again by (18), implies that $\mu_{n-3} \leq 1$ and hence that $\mu \subseteq \nu_{n-2}$. Hence $\theta$ (the outer border strip of $\nu_{n} \backslash \mu$ ) is almost Dyck, $\theta_{S} \subseteq \nu_{n} \backslash \mu$ and $\left|\theta_{S} \backslash \theta\right|=n-1$ is even. Furthermore, $\left(\nu_{n} \backslash \mu\right) \backslash \theta_{S}=\nu_{n-2} \backslash \mu$ and this is $s$-Dyck by induction. Hence $\nu_{n} \backslash \mu$ is $s$-Dyck, as desired.

Lemma 3.9. Let $n \in \mathbf{P}, n \geq 3$, and $\mu$ be a shifted partition such that $\mu \subseteq \delta_{n}^{-}$ $(\stackrel{\text { def }}{=}(n, n-1, \ldots, 3,2))$. Then $\delta_{n}^{-} \backslash \mu$ is $s$-Dyck if and only if there exist $2 \leq i_{1}<$ $\cdots<i_{r} \leq\left\lfloor\frac{n-1}{2}\right\rfloor\left(0 \leq r \leq\left\lfloor\frac{n-3}{2}\right\rfloor\right)$ and $(a, b) \in\{(3,2),(3,1),(1,0),(2,0)\}$ such that

$$
\mu= \begin{cases}\left(2 i_{r}+1,2 i_{r}, \ldots, 2 i_{1}+1,2 i_{1}, a, b\right), & \text { if } n \text { is odd }, \\ \left(n, 2 i_{r}+1,2 i_{r}, \ldots, 2 i_{1}+1,2 i_{1}, a, b\right), & \text { if } n \text { is even } .\end{cases}
$$

In this case, $d p\left(\delta_{n}^{-} \backslash \mu\right)=\left\lceil\frac{n-l(\mu)}{2}\right\rceil+d p((3,2) \backslash(a, b))-1$.

Proof. We proceed by induction on $n$. If $n \leq 4$, the result is easy to verify. Let $\mu=\left(\mu_{1}, \mu_{2}, \ldots, \mu_{l}\right)$.

Suppose that $\delta_{n}^{-} \backslash \mu$ is $s$-Dyck. If $\mu_{1}=n$, then $\delta_{n}^{-} \backslash \mu \approx \delta_{n-1}^{-} \backslash\left(\mu_{2}, \ldots, \mu_{l}\right)$ and by induction the result holds. If $\mu_{1}<n$, then since $\delta_{n}^{-} \backslash \mu$ is $s$-Dyck, $\mu_{1} \leq n-2$, so the outer border strip $\theta$ of $\delta_{n}^{-} \backslash \mu$ is a cbs of cardinality $n$ such that $l v(i)=l v(1)+i-1$ for $i=1, \ldots, n-1$, and $\operatorname{lv}(n)=l v(n-1)-1$. Therefore, since $\delta_{n}^{-} \backslash \mu$ is $s$-Dyck, we deduce that $n$ is odd and $\mu \subseteq \delta_{n-2}^{-}$. But $\delta_{n-2}^{-} \backslash \mu=\left(\delta_{n}^{-} \backslash \mu\right) \backslash \theta_{S}$, so the result follows by induction.

Conversely, suppose $\mu$ is of the form (19). If $\mu_{1}=n$, then $\delta_{n}^{-} \backslash \mu \approx \delta_{n-1}^{-} \backslash$ $\left(\mu_{2}, \ldots, \mu_{l}\right)$ and we are done by induction. If $\mu_{1}<n$, then, by (19), $n$ is odd, so $\mu_{1} \leq n-2$ and hence $\mu_{n-4} \leq 3$. Therefore, again by (19), $\mu_{n-2}=0$. Hence $\theta$ is almost Dyck, $\theta_{S} \subseteq \delta_{n}^{-} \backslash \mu$ and $\left|\theta_{S} \backslash \theta\right|=n-1$ is even, and $\mu \subseteq \delta_{n-2}^{-}$. But $\left(\delta_{n}^{-} \backslash \mu\right) \backslash \theta_{S}=\delta_{n-2}^{-} \backslash \mu$ is $s$-Dyck by induction, so $\delta_{n}^{-} \backslash \mu$ is $s$-Dyck, as desired.

\section{Parabolic Kazhdan-Lusztig polynomials}

In this section, using the results in the previous section and some from [5, we prove the main result of this work. More precisely, we give explicit combinatorial formulas for the parabolic Kazhdan-Lusztig polynomials of Hermitian symmetric pairs. Our results imply that these polynomials are combinatorial invariants.

We begin with the Hermitian symmetric pairs $\left(S_{n}^{B}, \mathcal{A}_{n-1}\right)$ and $\left(S_{n}^{D}, \mathcal{A}_{n-1}\right)$. Let $W^{J} \in\left\{\left(S_{n}^{B}\right)^{\mathcal{A}_{n-1}},\left(S_{n+1}^{D}\right)^{\mathcal{A}_{n}}\right\}$ and $u \in W^{J}$. By (2), (4), and the comments following Proposition 2.5 we may associate with $u$ a lattice path, which we will denote, for simplicity, by $\Lambda(u)$, with $(1,1)$ and $(1,-1)$ steps, starting at $(0,0)$ and having $n$ steps. Furthermore, by Propositions 2.5] and 2.9. we have that, for all $u, v \in W^{J}$, $u \leq v$ if and only if $\Lambda(u)$ lies (weakly) below $\Lambda(v)$ (write $\Lambda(u) \leq \Lambda(v)$ if this is the case). Recall the identification between lattice paths and shifted partitions made before Proposition 2.6. 
Theorem 4.1. Let $W^{J} \in\left\{\left(S_{n}^{B}\right)^{\mathcal{A}_{n-1}},\left(S_{n}^{D}\right)^{\mathcal{A}_{n-1}}\right\}$ and $u, v \in W^{J}, u \leq v$. Then

$$
P_{u, v}^{J, q}(q)=\left\{\begin{array}{l}
q^{\frac{1}{2}(|\lambda \backslash \mu|-d p(\lambda \backslash \mu))}, \quad \text { if } \lambda \backslash \mu \text { is } s-D y c k, \\
0, \text { otherwise, }
\end{array}\right.
$$

where $\mu \stackrel{\text { def }}{=} \Lambda(u), \lambda \stackrel{\text { def }}{=} \Lambda(v)$.

Proof. We proceed by induction on $l(v)=|\Lambda(v)|$, the result being clearly true if $v=e$. Let, for notational simplicity, $P_{x, y}^{J} \stackrel{\text { def }}{=} P_{x, y}^{J, q}(q)$ for all $x, y \in W^{J}$. We may clearly assume that $l(u, v) \geq 2$.

Assume first that $W^{J}=\left(S_{n}^{B}\right)^{\mathcal{A}_{n-1}}$ (so $\Lambda(z)=\Lambda_{B}(z)$ for all $z \in W^{J}$ ).

Suppose that $\lambda \backslash \mu$ has an upper peak $x$ and let $s=s_{i} \in S_{B} \backslash\left\{s_{0}\right\}$ be the corresponding element of $D(v)$ (see Lemma 2.7). Note that $\Lambda_{B}(v s)=\lambda \backslash\{x\}$. Then we have from Proposition 2.2 that

$$
P_{u, v}^{J}=\tilde{P}-\sum_{\{u \leq w<v s: w s<w\}} \mu(w, v s) q^{\frac{l(w, v)}{2}} P_{u, w}^{J},
$$

where

$$
\tilde{P}= \begin{cases}P_{u s, v s}^{J}+q P_{u, v s}^{J}, & \text { if } u s<u, \\ q P_{u s, v s}^{J}+P_{u, v s}^{J}, & \text { if } u<u s \in W^{J}, \\ 0, & \text { if } u<u s \notin W^{J} .\end{cases}
$$

Now let $w \in W^{J}$ be such that $u \leq w<v s$ and $w s<w$, and $\nu \stackrel{\text { def }}{=} \Lambda_{B}(w)$. Then from Lemma 2.7 we have that $\nu$ has an upper peak, $y$, below $x$. Furthermore, $y \neq x$ since $w \leq v s$. Note that this implies that $x$ is not the leftmost cell of $\lambda \backslash \mu$. Now, since $l(v s)<l(v)$, we conclude from our induction hypothesis that if $\mu(w, v s) \neq 0$, then $(\lambda \backslash\{x\}) \backslash \nu$ is $s$-Dyck and $d p((\lambda \backslash\{x\}) \backslash \nu)=1$. This implies that $d_{\lambda \backslash \nu}(x) \in\{2,3\}$. But $x^{(1)}$ (the cell directly below $x$ ) is an upper valley of $(\lambda \backslash\{x\}) \backslash \nu$, and $x^{(2)}$ (the cell directly above $y$ ) is a lower peak of $(\lambda \backslash\{x\}) \backslash \nu$ (possibly $\left.x^{(1)}=x^{(2)}\right)$. Therefore $d_{(\lambda \backslash \nu) \backslash\{x\}}(x-1)=d_{(\lambda \backslash \nu) \backslash\{x\}}\left(x^{(1)}\right)+1$, and this contradicts the fact that $(\lambda \backslash \nu) \backslash\{x\}$ is $s$-Dyck and $d p((\lambda \backslash \nu) \backslash\{x\})=1$. This shows that $\mu(w, v s)=0$ and hence that the sum on the right-hand side of (20) is also equal to zero. Therefore

$$
P_{u, v}^{J}= \begin{cases}P_{u s, v s}^{J}+q P_{u, v s}^{J}, & \text { if } u s<u, \\ q P_{u s, v s}^{J}+P_{u, v s}^{J}, & \text { if } u<u s \in W^{J}, \\ 0, & \text { if } u<u s \notin W^{J} .\end{cases}
$$

There are now three cases to consider.

a) $u<u s \notin W^{J}$.

Then $u(i) u(i+1)>0$ and this, by Proposition 2.6. implies that $\lambda \backslash \mu$ has neither a lower valley nor a lower peak below $x$. But then, by Proposition $3.4, \lambda \backslash \mu$ is not $s$-Dyck and the result follows from (21).

b) $u<u s \in W^{J}$.

Then $u(i)<0<u(i+1)$ and this, by Proposition 2.6. implies that $\lambda \backslash \mu$ has a lower valley, $y$, below $x$ and, clearly, $\Lambda_{B}(u s)=\mu \cup\{y\}$.

Suppose that $\lambda \backslash \mu$ is not $s$-Dyck. Then, by Theorem 3.5, we conclude that $(\lambda \backslash \mu) \backslash\{x, y\}$ and $(\lambda \backslash \mu) \backslash\{x\}$ are not $s$-Dyck. Hence we conclude from (21) and our induction hypothesis that $P_{u, v}^{J}=0$, as desired.

Suppose now that $\lambda \backslash \mu$ is $s$-Dyck. Then from Theorem 3.5 we conclude that exactly one of $(\lambda \backslash \mu) \backslash\{x, y\}$ and $(\lambda \backslash \mu) \backslash\{x\}$ is $s$-Dyck. If $(\lambda \backslash \mu) \backslash\{x, y\}$ is $s$-Dyck, 
then from Theorem 3.5, (21), and our induction hypothesis we conclude that

$$
\begin{aligned}
P_{u, v}^{J} & =q q^{\frac{1}{2}(|(\lambda \backslash \mu) \backslash\{x, y\}|-d p((\lambda \backslash \mu) \backslash\{x, y\}))} \\
& =q q^{\frac{1}{2}(|\lambda \backslash \mu|-2-d p(\lambda \backslash \mu))} \\
& =q^{\frac{1}{2}(|\lambda \backslash \mu|-d p(\lambda \backslash \mu))}
\end{aligned}
$$

as desired. If $(\lambda \backslash \mu) \backslash\{x\}$ is $s$-Dyck, then we conclude similarly that

$$
\begin{aligned}
P_{u, v}^{J} & =q^{\frac{1}{2}(|(\lambda \backslash \mu) \backslash\{x\}|-d p((\lambda \backslash \mu) \backslash\{x\}))} \\
& =q^{\frac{1}{2}(|\lambda \backslash \mu|-1-(d p(\lambda \backslash \mu)-1))} \\
& =q^{\frac{1}{2}(|\lambda \backslash \mu|-d p(\lambda \backslash \mu))}
\end{aligned}
$$

as desired.

c) $u>u s$.

Then, by Lemma 2.7 $\lambda \backslash \mu$ has a lower peak below $x$. Let $y$ be the cell directly below this lower peak. Then, clearly, $\Lambda_{B}(u s)=\mu \backslash\{y\}$.

Suppose that $\lambda \backslash \mu$ is not $s$-Dyck. Then, by Theorem 3.5 and Proposition 3.6. applied to $\eta=\lambda \backslash(\mu \backslash\{y\})=(\lambda \backslash \mu) \cup\{y\}, \eta \backslash\{x, y\}=(\lambda \backslash \mu) \backslash\{x\}$ and $\eta \backslash\{x\}=((\lambda \backslash \mu) \backslash\{x\}) \cup\{y\}$ are not $s$-Dyck. Hence we conclude from (21) and our induction hypothesis that $P_{u, v}^{J}=0$, as desired.

Suppose now that $\lambda \backslash \mu$ is $s$-Dyck. Then, again from Theorem 3.5, we conclude that exactly one of $(\lambda \backslash \mu) \backslash\{x\}$ and $((\lambda \backslash \mu) \backslash\{x\}) \cup\{y\}$ is s-Dyck. If $(\lambda \backslash \mu) \backslash\{x\}$ is $s$-Dyck, then from (21), Theorem 3.5, and our induction hypothesis we conclude that

$$
\begin{aligned}
P_{u, v}^{J} & =q q^{\frac{1}{2}(|(\lambda \backslash \mu) \backslash\{x\}|-d p((\lambda \backslash \mu) \backslash\{x\}))} \\
& =q q^{\frac{1}{2}(|(\lambda \backslash \mu)|-1-d p((\lambda \backslash \mu) \cup\{y\}))} \\
& =q^{\frac{1}{2}(|\lambda \backslash \mu|-d p(\lambda \backslash \mu))}
\end{aligned}
$$

as desired. If $((\lambda \backslash \mu) \backslash\{x\}) \cup\{y\}$ is $s$-Dyck, then we conclude similarly that

$$
\begin{aligned}
P_{u, v}^{J} & =q^{\frac{1}{2}(|((\lambda \backslash \mu) \backslash\{x\}) \cup\{y\}|-d p(((\lambda \backslash \mu) \backslash\{x\}) \cup\{y\}))} \\
& =q^{\frac{1}{2}(|\lambda \backslash \mu|-d p(\lambda \backslash \mu))}
\end{aligned}
$$

and the result again follows.

Suppose now that $\lambda \backslash \mu$ has no upper peaks. Let $\mu=\left(\mu_{1}, \ldots, \mu_{l}\right)$. Then (the lattice paths corresponding to) $\lambda$ and $\mu$ coincide from $(0,0)$ up to a certain point $P_{0}=(r+s, r-s)$, and to the right of this point all the steps of $\lambda$ are up. So $\lambda \backslash \mu \approx \delta_{n-r-s} \backslash \widetilde{\mu}$, where $\widetilde{\mu}=\left(\mu_{r+1}, \ldots, \mu_{l}\right)$. In particular, by Lemma 2.7, $s_{0} \in$ $D(v)$. Note that $\Lambda_{B}\left(v s_{0}\right)=\lambda_{-}$(same as $\lambda$, but with the last step down).

Let $w \in W^{J}$ be such that $u \leq w<v s_{0}$ and $w s_{0}<w$, and $\nu \stackrel{\text { def }}{=} \Lambda_{B}(w)$. Then from Lemma 2.7 we have that the last step of $\nu$ is up. Since $l\left(v s_{0}\right)<l(v)$, we conclude from our induction hypothesis that if $\mu\left(w, v s_{0}\right) \neq 0$, then $\lambda_{-} \backslash \nu$ is $s$-Dyck and $d p\left(\lambda_{-} \backslash \nu\right)=1$. This implies that the vertical distance between the (right end of the) $n$-th step of $\lambda_{-}$and the (right end of the) $n$-th step of $\nu$ is zero. This, since $\lambda_{-} \backslash \nu$ is $s$-Dyck and $d p\left(\lambda_{-} \backslash \nu\right)=1$, implies that $\left|\lambda_{-} \backslash \nu\right|=1$. This shows that $\nu$ coincides with $\lambda_{-}$except that its $(n-1)$-th and $n$-th steps are down and up, respectively, rather than up and down. Therefore $w=v s_{0} s_{1}$. This shows that 
$\mu\left(w, v s_{0}\right)=0$ unless $w=v s_{0} s_{1}$. Therefore

$$
\sum_{\left\{u \leq w<v s_{0}: w s_{0}<w\right\}} \mu\left(w, v s_{0}\right) q^{\frac{l(w, v)}{2}} P_{u, w}^{J}=q P_{u, v s_{0} s_{1}}^{J}
$$

(note that $u \leq v s_{0} s_{1}$ since $|\lambda \backslash \mu| \geq 2$ ). Hence, since $u s_{0} \in W^{J}$, we conclude from Proposition 2.2 that

$$
P_{u, v}^{J}= \begin{cases}P_{u s_{0}, v s_{0}}^{J}+q P_{u, v s_{0}}^{J}-q P_{u, v s_{0} s_{1}}^{J}, & \text { if } u s_{0}<u, \\ q P_{u s_{0}, v s_{0}}^{J}+P_{u, v s_{0}}^{J}-q P_{u, v s_{0} s_{1}}^{J}, & \text { if } u<u s_{0} .\end{cases}
$$

We now distinguish two cases.

i) $u s_{0}<u$.

Then, by Proposition 2.4, $u(1)<0$ and, by Lemma 2.7, the last step of $\mu$ is up. So $\Lambda_{B}\left(u s_{0}\right)=\mu_{-}$, and the last part of $\mu$ is 1 .

Suppose $\lambda \backslash \mu$ is $s$-Dyck. Then, by Lemmas 3.7 and 3.8, $\nu \backslash \mu$ is not $s$-Dyck (where $\nu \stackrel{\text { def }}{=} \Lambda_{B}\left(v s_{0} s_{1}\right)$ ) and $l(\widetilde{\mu}) \equiv n-r-s(\bmod 2)$. Furthermore, by Lemmas 3.7 and 3.9, $\lambda_{-} \backslash \mu$ is $s$-Dyck if and only if $\mu_{l-1} \geq 4$ while $\lambda_{-} \backslash \mu_{-}$is $s$-Dyck if and only if $\mu_{l-1}=2$. Therefore we conclude from (22) and our induction hypothesis that

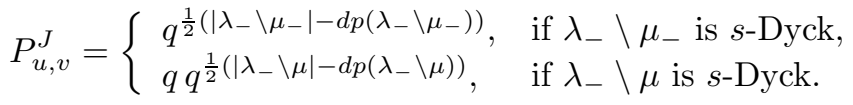

But, by Lemmas 3.7 and 3.9 .

$$
d p\left(\lambda_{-} \backslash \mu_{-}\right)=\left\lceil\frac{n-r-s-l(\tilde{\mu})+1}{2}\right\rceil-1=d p(\lambda \backslash \mu)
$$

if $\lambda_{-} \backslash \mu_{-}$is $s$-Dyck, while

$$
d p\left(\lambda_{-} \backslash \mu\right)=\left\lceil\frac{n-r-s-l(\tilde{\mu})}{2}\right\rceil+d p((3,2) \backslash(1,0))-1=1+d p(\lambda \backslash \mu)
$$

if $\lambda_{-} \backslash \mu$ is $s$-Dyck, so the result holds in this case.

Suppose now that $\lambda \backslash \mu$ is not $s$-Dyck. If $\nu \backslash \mu$ is not $s$-Dyck, then, by Lemmas 3.8 and 3.9, $\lambda_{-} \backslash \mu$ and $\lambda_{-} \backslash \mu_{-}$are not $s$-Dyck, and the result follows from (22) and our induction hypothesis. So assume $\nu \backslash \mu$ is $s$-Dyck. Then it follows from Lemmas 3.8 and 3.9 that $l(\widetilde{\mu}) \not \equiv n-r-s(\bmod 2)$ and that $\lambda_{-} \backslash \mu$ is $s$-Dyck if and only if $\mu_{l-1}=3$ while $\lambda_{-} \backslash \mu_{-}$is $s$-Dyck if and only if $\mu_{l-1}=2$.

If $\lambda_{-} \backslash \mu$ is $s$-Dyck, then we conclude from Lemmas 3.9 and 3.8 that

$$
d p(\nu \backslash \mu)=\left\lceil\frac{n-r-s-l(\tilde{\mu})}{2}\right\rceil-1=d p\left(\lambda_{-} \backslash \mu\right)-1 .
$$

Therefore, by our induction hypothesis, $P_{u, v s_{0} s_{1}}^{J}=q^{\frac{1}{2}(|\nu \backslash \mu|-d p(\nu \backslash \mu))}=P_{u, v s_{0}}^{J}$, and the result follows from (22).

Similarly, if $\lambda_{-} \backslash \mu_{-}$is $s$-Dyck, then we conclude that

$$
\begin{aligned}
d p(\nu \backslash \mu) & =\left\lceil\frac{n-r-s-l(\tilde{\mu})}{2}\right\rceil+d p((3,1) \backslash(2,1))-1 \\
& =\left\lceil\frac{n-r-s-l(\tilde{\mu})+1}{2}\right\rceil+d p((3,2) \backslash(2,0))-1 \\
& =d p\left(\lambda_{-} \backslash \mu_{-}\right) .
\end{aligned}
$$


Therefore, by induction, $q P_{u, v s_{0} s_{1}}^{J}=q q^{\frac{1}{2}(|\nu \backslash \mu|-d p(\nu \backslash \mu))}=P_{u s_{0}, v s_{0}}^{J}$, and the result follows from (22).

ii) $u<u s_{0}$.

Then, by Proposition 2.4, $u(1)>0$ and hence, by Proposition 2.6, the last step of $\mu$ is down. So $\mu_{l} \geq 2$, and $\Lambda_{B}\left(u s_{0}\right)=\mu_{+}$.

Suppose $\lambda \backslash \mu$ is $s$-Dyck. Then, by Lemmas 3.7 and 3.8, $\nu \backslash \mu$ is not $s$-Dyck, and $l(\widetilde{\mu}) \not \equiv n-r-s(\bmod 2)$. Furthermore, by Lemmas 3.7 and 3.9, $\lambda_{-} \backslash \mu$ is $s$-Dyck if and only if $\mu_{l}=2$ while $\lambda_{-} \backslash \mu_{+}$is $s$-Dyck if and only if $\mu_{l} \geq 4$. Therefore we conclude from (22) and our induction hypothesis that

$$
P_{u, v}^{J}= \begin{cases}q^{\frac{1}{2}\left(\left|\lambda_{-} \backslash \mu\right|-d p\left(\lambda_{-} \backslash \mu\right)\right)}, & \text { if } \lambda_{-} \backslash \mu \text { is } s \text {-Dyck } \\ q q^{\frac{1}{2}\left(\left|\lambda_{-} \backslash \mu_{+}\right|-d p\left(\lambda_{-} \backslash \mu_{+}\right)\right)}, & \text {if } \lambda_{-} \backslash \mu_{+} \text {is } s \text {-Dyck. }\end{cases}
$$

But, by Lemmas 3.7 and 3.9 .

$$
d p\left(\lambda_{-} \backslash \mu\right)=\left\lceil\frac{n-r-s-l(\tilde{\mu})}{2}\right\rceil-1=d p(\lambda \backslash \mu)-1
$$

if $\mu_{l}=2$, while

$$
\begin{aligned}
d p\left(\lambda_{-} \backslash \mu_{+}\right) & =\left\lceil\frac{n-r-s-l(\tilde{\mu})-1}{2}\right\rceil+d p((3,2) \backslash(1,0))-1 \\
& =\left\lceil\frac{n-r-s-l(\tilde{\mu})-2}{2}\right\rceil+1 \\
& =d p(\lambda \backslash \mu)
\end{aligned}
$$

if $\mu_{l} \geq 4$, so the result holds in this case.

Suppose now that $\lambda \backslash \mu$ is not $s$-Dyck. If $\nu \backslash \mu$ is not $s$-Dyck, then, by Lemmas 3.8 and 3.9. $\lambda_{-} \backslash \mu$ and $\lambda_{-} \backslash \mu_{+}$are not $s$-Dyck, and the result follows from (22) and our induction hypothesis. So assume that $\nu \backslash \mu$ is $s$-Dyck. Then it follows from Lemmas 3.8 and 3.9 that $l(\widetilde{\mu}) \equiv n-r-s(\bmod 2)$ and that $\lambda_{-} \backslash \mu$ is $s$-Dyck if and only if $\mu_{l}=2$ while $\lambda_{-} \backslash \mu_{+}$is $s$-Dyck if and only if $\mu_{l}=3$. Furthermore,

$$
\begin{aligned}
d p(\nu \backslash \mu) & =\left\lceil\frac{n-r-s-l(\tilde{\mu})}{2}\right\rceil+d p((3,1) \backslash(2,0))-1 \\
& =\left\lceil\frac{n-r-s-l(\tilde{\mu})}{2}\right\rceil+1 \\
& =d p\left(\lambda_{-} \backslash \mu\right)+1
\end{aligned}
$$

if $\mu_{l}=2$, while

$$
\begin{aligned}
d p(\nu \backslash \mu) & =\left\lceil\frac{n-r-s-l(\tilde{\mu})}{2}\right\rceil+d p((3,1) \backslash(3,0))-1 \\
& =\left\lceil\frac{n-r-s-l(\tilde{\mu})-1}{2}\right\rceil \\
& =d p\left(\lambda_{-} \backslash \mu_{+}\right)
\end{aligned}
$$

if $\mu_{l}=3$, so the result follows from (22) and our induction hypothesis.

Assume now that $W^{J}=\left(S_{n}^{D}\right)^{\mathcal{A}_{n-1}}$.

If $\lambda \backslash \mu$ has an upper peak, then the proof is analogous to that of the corresponding case for $\left(S_{n}^{B}\right)^{\mathcal{A}_{n-1}}$ (except that $s \in S_{D} \backslash\left\{\tilde{s}_{0}, s_{1}\right\}$ ).

So suppose that $\lambda \backslash \mu$ has no upper peaks. Let $\mu=\left(\mu_{1}, \ldots, \mu_{l}\right)$. Then (the paths corresponding to) $\lambda$ and $\mu$ coincide from $(0,0)$ up to a certain point $P_{0}=(r+s, r-s)$, 
and to the right of this point all the steps of $\lambda$ are up. So $\lambda \backslash \mu \approx \delta_{n-1-r-s} \backslash \tilde{\mu}$, where $\widetilde{\mu} \stackrel{\text { def }}{=}\left(\mu_{r+1}, \ldots, \mu_{l}\right)$. In particular, by Lemma 2.11, there exists $s \in\left\{\tilde{s}_{0}, s_{1}\right\}$ such that $s \in D(v)$ (more precisely, $s=\tilde{s}_{0}$ if $\lambda$ has an odd number of up-steps and $s=s_{1}$ if $\lambda$ has an even number of up-steps). Furthermore, $\Lambda_{D}(v s)=\lambda_{-}$.

Let $w \in W^{J}$ be such that $u \leq w<v s$ and $w s<w$, and $\nu \stackrel{\text { def }}{=} \Lambda_{D}(w)$. Then from Lemma 2.11 we have that the last step of $\nu$ is up and the number of upsteps of $\nu$ is of the same parity as that of $\lambda$. Since $l(v s)<l(v)$, we conclude from our induction hypothesis that if $\mu(w, v s) \neq 0$, then $\lambda_{-} \backslash \nu$ is $s$-Dyck and $d p\left(\lambda_{-} \backslash \nu\right)=1$. This implies that the vertical distance between the (right end of the) $(n-1)$-th step of $\lambda_{-}$and the (right end of the) $(n-1)$-th step of $\nu$ is zero (else $d_{\lambda_{-} \backslash \nu}(n-2)>d_{\lambda_{-} \backslash \nu}(n-1)>0$, which contradicts the fact that $\lambda_{-} \backslash \nu$ is $s$-Dyck and $\left.d p\left(\lambda_{-} \backslash \nu\right)=1\right)$. But this implies that $\nu$ has a number of up-steps which is of different parity than that of $\lambda$, which is a contradiction. This shows that $\mu(w, v s)=0$. Therefore

$$
\sum_{\{u \leq w<v s: w s<w\}} \mu(w, v s) q^{\frac{l(w, v)}{2}} P_{u, w}^{J}=0 .
$$

Hence, we conclude from Proposition 2.2 that

$$
P_{u, v}^{J}= \begin{cases}P_{u s, v s}^{J}+q P_{u, v s}^{J}, & \text { if } u s<u, \\ q P_{u s, v s}^{J}+P_{u, v s}^{J}, & \text { if } u<u s \in W^{J}, \\ 0, & \text { if } u<u s \notin W^{J} .\end{cases}
$$

There are now three cases to consider.

i) $u<u s \notin W^{J}$.

Assume that $\lambda \backslash \mu$ is $s$-Dyck.

If $u(2)>0$, then, by Proposition 2.10, the last $((n-1)$-th) step of $\mu$ is down. Furthermore, since $u \in W^{J}, u(1)<0$ if $s=\tilde{s}_{0}$ while $u(1)>0$ if $s=s_{1}$. Hence, by Proposition 2.10, $\mu$ has an odd number of up-steps if $s=\tilde{s}_{0}$ and an even number of up-steps if $s=s_{1}$. Hence the last part of $\mu$ is $\geq 2$ and $\mu$ has a number of up-steps of the same parity as that of $\lambda$. Therefore $l(\widetilde{\mu}) \equiv n-1-r-s(\bmod 2)$, and this contradicts Lemma 3.7 .

If $u(2)<0$, then the last step of $\mu$ is up and $\mu$ has a number of up-steps of different parity than that of $\lambda$. Hence $\mu_{l}=1$ and $l(\widetilde{\mu}) \not \equiv n-1-r-s(\bmod 2)$. This, by Lemma 3.7, is a contradiction.

Hence $\lambda \backslash \mu$ is not $s$-Dyck, and the result follows in this case.

ii) $u<u s \in W^{J}$.

Then, since $u \in W^{J}, u(2)>0$. Hence the last step of $\mu$ is down and reasoning as above we conclude that $\mu$ has a number of up-steps of different parity than that of $\lambda$. Therefore, the last part of $\mu$ is $\geq 2$ and $l(\widetilde{\mu}) \not \equiv n-1-r-s(\bmod 2)$. Also, $\Lambda_{D}(u s)=\mu_{+}$.

Suppose $\lambda \backslash \mu$ is $s$-Dyck. Then, by Lemmas 3.7 and 3.9, $\lambda_{-} \backslash \mu$ is $s$-Dyck if and only if $\mu_{l}=2$ while $\lambda_{-} \backslash \mu_{+}$is $s$-Dyck if and only if $\mu_{l} \geq 4$. Therefore we conclude from (23) and our induction hypothesis that

$$
P_{u, v}^{J}= \begin{cases}q^{\frac{1}{2}\left(\left|\lambda_{-} \backslash \mu\right|-d p\left(\lambda_{-} \backslash \mu\right)\right)}, & \text { if } \lambda_{-} \backslash \mu \text { is } s \text {-Dyck } \\ q q^{\frac{1}{2}\left(\left|\lambda_{-} \backslash \mu_{+}\right|-d p\left(\lambda_{-} \backslash \mu_{+}\right)\right)}, & \text {if } \lambda_{-} \backslash \mu_{+} \text {is } s \text {-Dyck. }\end{cases}
$$


But, by Lemmas 3.7 and 3.9 .

$$
d p\left(\lambda_{-} \backslash \mu\right)=d p(\lambda \backslash \mu)-1
$$

if $\mu_{l}=2$, while

$$
\begin{aligned}
d p\left(\lambda_{-} \backslash \mu_{+}\right) & =\left\lceil\frac{n-1-r-s-l(\tilde{\mu})-1}{2}\right\rceil+d p((3,2) \backslash(1,0))-1 \\
& =\left\lceil\frac{n-1-r-s-l(\tilde{\mu})}{2}\right\rceil \\
& =d p(\lambda \backslash \mu)
\end{aligned}
$$

if $\mu_{l} \geq 4$, so the result holds in this case.

Suppose now that $\lambda \backslash \mu$ is not $s$-Dyck. Then $\lambda_{-} \backslash \mu$ is not $s$-Dyck (else, by Lemma 3.9, $\tilde{\mu}$ would be almost even), and $\lambda_{-} \backslash \mu_{+}$is not $s$-Dyck (else, by Lemma 3.9, $\widetilde{\mu}_{+}$ would be almost even), so, by induction, the result again holds.

iii) $u s<u$.

Then, by Lemma 2.11, the last step of $\mu$ is up and $\mu$ has a number of up-steps of the same parity as that of $\lambda$, while $\Lambda_{D}(u s)=\mu_{-}$. Hence $\mu_{l}=1$ and $l(\widetilde{\mu}) \equiv n-1-r-s$ $(\bmod 2)$.

Suppose $\lambda \backslash \mu$ is $s$-Dyck. Then, by Lemmas 3.7 and 3.9, $\lambda_{-} \backslash \mu$ is $s$-Dyck if and only if $\mu_{l-1} \geq 4$ while $\lambda_{-} \backslash \mu_{-}$is $s$-Dyck if and only if $\mu_{l-1}=2$. Therefore we conclude from (23) and our induction hypothesis that

$$
P_{u, v}^{J}= \begin{cases}q^{\frac{1}{2}\left(\left|\lambda_{-} \backslash \mu_{-}\right|-d p\left(\lambda_{-} \backslash \mu_{-}\right)\right),} & \text {if } \lambda_{-} \backslash \mu_{-} \text {is } s \text {-Dyck, } \\ q q^{\frac{1}{2}\left(\left|\lambda_{-} \backslash \mu\right|-d p\left(\lambda_{-} \backslash \mu\right)\right),} & \text { if } \lambda_{-} \backslash \mu \text { is } s \text {-Dyck. }\end{cases}
$$

But, by Lemma 3.9.

$$
d p\left(\lambda_{-} \backslash \mu_{-}\right)=\left\lceil\frac{n-1-r-s-l(\tilde{\mu})+1}{2}\right\rceil-1=d p(\lambda \backslash \mu)
$$

if $\lambda_{-} \backslash \mu_{-}$is $s$-Dyck, while

$$
d p\left(\lambda_{-} \backslash \mu\right)=\left\lceil\frac{n-1-r-s-l(\tilde{\mu})}{2}\right\rceil+d p((3,2) \backslash(1,0))-1=1+d p(\lambda \backslash \mu)
$$

if $\lambda_{-} \backslash \mu$ is $s$-Dyck, so the result holds in this case.

Suppose now that $\lambda \backslash \mu$ is not $s$-Dyck. Then $\lambda_{-} \backslash \mu$ is not $s$-Dyck (else, by Lemma 3.9. $\mu$ would be almost even) and $\lambda_{-} \backslash \mu_{-}$is not $s$-Dyck (else, by Lemma 3.9 $\mu$ would be almost even), so, by (23) and our induction hypothesis, the result again holds.

This concludes the induction step and hence the proof.

We illustrate the preceding theorem with an example. Let $W^{J}=\left(S_{6}^{B}\right)^{\mathcal{A}_{5}}, u=$ $[-2,3,4,-1,5,6], v=[-4,-3,5,-2,-1,6]$. Then $\Lambda_{B}(u)=(4,1)$ and $\Lambda_{B}(v)=$ $(5,4,2,1)$, so, by Theorem 4.1 and our definitions, $P_{u, v}^{J, q}(q)=q^{\frac{1}{2}(7-1)}=q^{3}$. On the other hand, if $W^{J}=\left(S_{6}^{D}\right)^{\mathcal{A}_{5}}$, then $\Lambda_{D}(u)=(3)$ and $\Lambda_{D}(v)=(4,3,1)$, so by Theorem 4.1 and our definitions $P_{u, v}^{J, q}(q)=0$.

We now consider the Hermitian symmetric pairs $\left(S_{n}^{B}, \mathcal{B}_{n-1}\right)$ and $\left(S_{n}^{D}, \mathcal{D}_{n-1}\right)$.

Theorem 4.2. Let $u, v \in\left(S_{n}^{B}\right)^{\mathcal{B}_{n-1}}, u \leq v$. Then

$$
P_{u, v}^{J, q}(q)= \begin{cases}1, & \text { if } l(u, v) \leq 1, \\ 0, & \text { otherwise. }\end{cases}
$$


Proof. We proceed by induction on $l(u, v)$, the result being clear if $l(u, v) \leq 1$. So assume that $l(u, v) \geq 2$. Then from [7, Proposition 3.1], [5, Theorem 3.2], [8, Corollary 2.2], and our induction hypothesis we have that

$$
q^{l(u, v)} P_{u, v}^{J, q}\left(\frac{1}{q}\right)-P_{u, v}^{J, q}(q)=(-1)^{l(u, c)}(1-q)+(-1)^{l(u, v)}(1-q),
$$

where $c$ is the only element of $[u, v]^{J}$ covered by $v$, and the result follows.

Recall the notation $v_{\varepsilon i}(\varepsilon \in\{-1,1\}, i \in[n])$ introduced in $\S 2$ for the elements of $\left(S_{n}^{D}\right)^{\mathcal{D}_{n-1}}$.

Theorem 4.3. Let $u, v \in\left(S_{n}^{D}\right)^{\mathcal{D}_{n-1}}, u<v, l(u, v) \geq 2$. Then

$$
P_{u, v}^{J, q}(q)= \begin{cases}q^{l(v)-n}, & \text { if } 1 \leq 2 n-l(u)-l(v) \leq 2, \\ 0, & \text { otherwise. }\end{cases}
$$

Proof. We proceed by induction on $l(u, v)$, the result being easy to check if $l(u, v)=$ 2. So assume $l(u, v) \geq 3$. If $[u, v]^{J}$ is a chain, then $2 n-l(u)-l(v) \notin\{1,2\}$ and the result follows as in Theorem 4.2 . If $[u, v]^{J}$ is not a chain, then there exists $i, j \in[2, n]$ such that $u=v_{i}$ and $v=v_{-j}$. Note that $2 n-l\left(v_{i}\right)-l\left(v_{-j}\right)=i+2-j$. Then from [7, Proposition 3.1], 5, Theorem 3.3], 8, Corollary 2.2], and our induction hypothesis we conclude that

$$
\begin{aligned}
q^{i+j-2} & P_{v_{i}, v_{-j}}^{J, q}\left(\frac{1}{q}\right)-P_{v_{i}, v_{-j}}^{J, q}(q) \\
& =\sum_{a=1}^{i-1} R_{v_{i}, v_{a}}^{J, q}(q) P_{v_{a}, v_{-j}}^{J, q}(q)+\sum_{b=1}^{j} R_{v_{i}, v_{-b}}^{J, q}(q) P_{v_{-b}, v_{-j}}^{J, q}(q) \\
& =\sum_{a \in[i-1] \cap\{j-1, j\}} R_{v_{i}, v_{a}}^{J, q}(q) q^{j-2}+R_{v_{i}, v_{-j}}^{J, q}(q)+R_{v_{i}, v_{-(j-1)}}^{J, q}(q) \\
& = \begin{cases}-(1-q)+(1-q)\left(1-q^{i-1}\right), & \text { if } j=i+1, \\
(q-1) q^{i-2}+(1-q)\left(1-q^{i-1}\right)+q-1, & \text { if } j=i, \\
0, & \text { otherwise, }\end{cases}
\end{aligned}
$$

and the result follows.

Note that from Proposition 2.3 we have that $P_{u, v}^{J,-1}(q)=P_{w_{0}(J) u, w_{0}(J) v}(q)$ for all $u, v \in W^{J}$ and all Hermitian symmetric pairs $\left(W, W_{J}\right)$. On the other hand, the polynomials $P_{w_{0}(J) u, w_{0}(J) v}(q)$ have been computed (for $u, v \in W^{J}$ and $\left(W, W_{J}\right)$ a Hermitian symmetric pair) in [3. Thus, the results in this section, together with the main result of 4, complete the computation of the parabolic Kazhdan-Lusztig polynomials of Hermitian symmetric pairs.

Corollary 4.4. Let $\left(W, W_{J}\right)$ be a Hermitian symmetric pair and $u, v \in W^{J}, u \leq v$. Then $P_{u, v}^{J, q}(q)$ is either zero or a monic power of $q$.

Proof. This follows immediately from Theorems 4.1 4.2. and 4.3. the main result of [4, §5], and computer calculations.

The parabolic Kazhdan-Lusztig polynomials for the exceptional Hermitian symmetric pairs have been computed by implementing in Maple 6 the recursions given by [7, Proposition 3.1]. These implementations used two Maple packages for handling finite Coxeter groups (Version 2.3) and posets (Version 2.2) developed by John Stembridge. 
In the case of lower intervals, more can be said.

Corollary 4.5. Let $\left(W, W_{J}\right)$ be a Hermitian symmetric pair and $v \in W^{J}$. If $P_{e, v}^{J, q}(q) \neq 0$, then

$$
P_{e, v}^{J, q}(q)=q^{\sum_{j \in T(v)}(j-1)},
$$

where $T(v)$ has the same meaning as in [5, Corollary 3.5].

Proof. If $W^{J}=\left(S_{n}\right)^{S_{B} \backslash\left\{s_{0}, s_{i}\right\}}$ for some $i \in[n-1]$, then the result follows immediately from Corollaries 5.2 and 3.5 of [4]. Assume now that $W^{J} \in\left\{\left(S_{n}^{B}\right)^{\mathcal{A}_{n-1} \text {, }}\right.$ $\left.\left(S_{n}^{D}\right)^{\mathcal{A}_{n-1}}\right\}$. Let $\lambda \stackrel{\text { def }}{=} \Lambda(v)$ (notation as in Theorem 4.1). We claim that $\lambda$ is $s$ Dyck if and only if $\lambda=\delta_{i}$ for some $i \equiv 1(\bmod 2)$. It follows immediately from our definitions that $\delta_{i}$ is $s$-Dyck if $i$ is odd, and that $d p\left(\delta_{i}\right)=\frac{i+1}{2}$. Conversely, suppose $\lambda=\left(\lambda_{1}, \lambda_{2}, \ldots\right)$ is $s$-Dyck. We proceed by induction on $\lambda_{1}$, our claim being easy to check if $\lambda_{1} \leq 2$. So suppose $\lambda_{1} \geq 3$. Let $\theta$ be the outer border strip of $\lambda$. Since $\lambda \backslash \theta_{S}$ is $s$-Dyck we conclude from our induction hypothesis that $\lambda \backslash \theta_{S}=\delta_{i}$ for some $i \equiv 1(\bmod 2)$. Therefore, since $\lambda$ is $s$-Dyck, $\lambda=\delta_{i+2}$. Hence, by Theorem 4.1 . we conclude that $\Lambda(v)=\delta_{i}$ for some $i$ odd, and the result follows from Corollary 3.7 of [5] and Theorem 4.1. Finally, for the other Hermitian symmetric pairs, the result follows immediately from Theorems 3.2 and 3.3 of [5], Theorems 4.2 and 4.3 and from computer calculations.

The results in this section, and simple examples, show that (as for the polynomials $R_{u, v}^{J, q}(q)$, see the comments following Corollary 3.5 of [5]) given a Hermitian symmetric pair $\left(W, W_{J}\right)$ and $u, v \in W^{J}$ there may not exist $w \in W^{J}$ such that $P_{u, v}^{J, q}(q)=P_{e, w}^{J, q}(q)$.

Let $w_{0}^{J}$ be the longest element of $W^{J}$. For a finite Coxeter group $W$, let $E(W) \subset$ $\mathbf{P}$ be its set of exponents.

Corollary 4.6. Let $\left(W, W_{J}\right)$ be a Hermitian symmetric pair. Then there exists $e \in E(W)$ such that, for all $v \in W^{J}$, if $P_{v, w_{0}^{J}}^{J, q}(q) \neq 0$, then

$$
P_{v, w_{0}^{J}}^{J, q}(q)=q^{(e-1) r}
$$

for some $r \in \mathbf{N}$.

Proof. If $W^{J}=\left(S_{n}\right)^{S_{B} \backslash\left\{s_{0}, s_{i}\right\}}$ for some $i \in[n-1]$, then the result follows directly from Corollary 4.4. Assume now that $W^{J} \in\left\{\left(S_{n}^{B}\right)^{\mathcal{A}_{n-1}},\left(S_{n+1}^{D}\right)^{\mathcal{A}_{n}}\right\}$. Let $\mu \stackrel{\text { def }}{=} \Lambda(v)$. By Theorem 4.1 we have that $P_{v, w_{0}^{J}}^{J, q}(q) \neq 0$ if and only if $\delta_{n} \backslash \mu$ is $s$-Dyck. But it is easy to see from the proof of Lemma 3.7. by induction on $n$, that if $\delta_{n} \backslash \mu$ is $s$-Dyck, then $\left|\delta_{n} \backslash \mu\right| \equiv d p\left(\delta_{n} \backslash \mu\right)(\bmod 4)$, and the result follows from Theorem 4.1 (with $e=3$ ). Finally, the result follows immediately from Theorems 4.2 and 4.3 and from computer calculations (with $e=4$ and $e=5$ ) for the other Hermitian symmetric pairs.

It is a long standing open problem in the theory of Kazhdan-Lusztig polynomials to decide if they depend only on the poset isomorphism class of the interval determined under Bruhat order by the elements that index them (see, e.g., 2, $\S 5.6$, p. 161]). It is therefore natural to ask the same question for the parabolic Kazhdan-Lusztig polynomials. We conclude this section by showing, using the previous results, that this is true for the parabolic Kazhdan-Lusztig polynomials of Hermitian symmetric pairs. 
We need first the following purely combinatorial result on skew shifted partitions. Let $\theta$ be a skew shifted partition and $E(\theta)$ be the set of all elements $x \in \theta$ such that $\theta \backslash\{x\}$ is connected, $|\{a \in \theta: a \triangleleft x\}| \leq 1$, and $|\{a \in \theta: a \triangleright x\}| \leq 1$. Note that $|E(\theta)|$ depends only on the isomorphism class of $\theta$ as a poset.

Lemma 4.7. Let $\theta, \eta$ be two connected skew shifted partitions that are isomorphic as posets, $|\theta| \geq 4$. Then:

i) either $\theta \approx \eta$ or $\theta \approx \eta^{\prime}$ if $|E(\theta)|=2$;

ii) $\theta \approx \eta$ if $|E(\theta)| \geq 3$.

Proof. Clearly, the leftmost and rightmost cells of $\theta$ are in $E(\theta)$. Let $x$ be a cell of $E(\theta)$ that is neither the leftmost cell, nor one of the rightmost cells, of $\theta$. Then necessarily

$$
|\{a \in \theta: a \triangleleft x\}|=|\{a \in \theta: a \triangleright x\}|=1 .
$$

Let $a$ (respectively, $b$ ) be the only element of $\theta$ that covers (respectively, is covered by) $x$. Then $a$ and $b$ must lie on opposite sides of $x$ (else $x$ would be either the leftmost cell, or one of the rightmost cells, of $\theta$ ). Hence $\theta \backslash\{x\}$ is not connected, which is a contradiction since $x \in E(\theta)$. This shows that $E(\theta)$ consists exactly of the leftmost and rightmost cells of $\theta$.

If $|E(\theta)|=2$, then $\theta$ is a finite convex subset of $\mathbf{P}^{2}$ (i.e., a skew partition) and the result follows from Lemma 5.5 of [4].

So assume $|E(\theta)| \geq 3$. Then $\theta$ has at least two rightmost cells. Let, for $y \in E(\theta)$, $n(y) \stackrel{\text { def }}{=} \mid\{x \in E(\theta) \backslash\{y\}: x$ is comparable to $y\} \mid$. Note that the rightmost cells of $\theta$ are a chain while the leftmost cell of $\theta$ is comparable to at most two rightmost cells of $\theta$. Therefore $n(1) \leq 2$ while $n(y) \geq|E(\theta)|-2$ if $y$ is any rightmost cell of $\theta$. There are now two cases to distinguish.

If there is a unique element $y \in E(\theta)$ such that

$$
n(y)=\min \{n(x): x \in E(\theta)\},
$$

then $y$ is the leftmost cell of $\theta$. Let $\left(a_{1}, b_{1}, \ldots, a_{r}, b_{r}\right),\left(c_{1}, d_{1}, \ldots, c_{s}, d_{s}\right)$ be the polygon notation of $\theta$ introduced in $\S 2$. Let $x_{1}$ be the maximal element of $\theta$ which is closest to $y$ (in the sense of the distance in the Hasse graph of $\theta$ ). Then the distance between $y$ and $x_{1}$ is $a_{1}-1$. Now let $y_{1}$ be the upper valley of $\theta$ which is closest to $x_{1}$. Then the distance between $x_{1}$ and $y_{1}$ is $b_{1}$. Now let $x_{2}$ be the closest element to $y_{1}$ among the maximal elements of $\theta$ which are different from $x_{1}$. Then the distance between $x_{2}$ and $y_{1}$ is $a_{2}$. Continuing in this way we obtain a sequence $x_{1}, y_{1}, \ldots, y_{r-1}, x_{r}$ of elements of $\theta$ such that $y_{1}, \ldots, y_{r-1}$ are all the upper valleys of $\theta$. Let $y_{r}$ be the element of $E(\theta) \backslash\{y\}$ which is closest to $x_{r}$. Then the distance between $x_{r}$ and $y_{r}$ is $b_{r}-1$. This shows that the sequence $\left(a_{1}, b_{1}, \ldots, a_{r}, b_{r}\right)$ depends only on the isomorphism class of $\theta$ as a poset. Similarly, we obtain the same conclusion for $\left(c_{1}, d_{1}, \ldots, c_{s}, d_{s}\right)$, and the result follows.

If there is more than one element $y$ such that (24) holds, then $4 \geq|E(\theta)|$. If $|E(\theta)|=3$, then either $\theta \approx(k, l)$ or $\theta \approx\left(\delta_{k} \backslash(k-1, k-2, \ldots, l+1, l-1, \ldots, 3,2,1)\right)$ for some $k>l>0, k \geq 3$. If $\theta$ has exactly one maximal and one minimal element, then $k=l+1$ and the result follows. Otherwise $|\theta|=k+l$ and $2(l-1)$ is the number of elements of $\theta$ of degree 3 , so $k$ and $l$ depend only on the isomorphism class of $\theta$ as a poset, and the result again follows. If $|E(\theta)|=4$, then $n(1)=2$ and $\theta \approx(3,2,1)$. Hence, in any case, we conclude that $\theta \approx \eta$. 
We can now prove that parabolic Kazhdan-Lusztig and $R$-polynomials of Hermitian symmetric pairs are combinatorial invariants. Recall the notation $\Lambda(u)$ defined before Theorem 4.1 for $u \in W^{J}$ and $W^{J} \in\left\{\left(S_{n}^{B}\right)^{\mathcal{A}_{n-1}},\left(S_{n}^{D}\right)^{\mathcal{A}_{n-1}}\right\}$, and the identification between shifted partitions and lattice paths pointed out after Proposition 2.5 .

Corollary 4.8. Let $\left(W, W_{J}\right)$ be a Hermitian symmetric pair, and $u, v, w, z \in W^{J}$ be such that $[u, v]^{J} \cong[w, z]^{J}$ (as posets). Then

$$
P_{u, v}^{J, q}(q)=P_{w, z}^{J, q}(q)
$$

(and hence also $R_{u, v}^{J, q}(q)=R_{w, z}^{J, q}(q), R_{u, v}^{J,-1}(q)=R_{w, z}^{J,-1}(q)$ and $P_{u, v}^{J,-1}(q)=P_{w, z}^{J,-1}(q)$ ).

Proof. If $W^{J}=\left(S_{n}\right)^{S_{B} \backslash\left\{s_{0}, s_{i}\right\}}$ for some $i \in[n-1]$, then the result follows from Corollary 5.6 of [4.

So assume that $W^{J} \in\left\{\left(S_{n}^{D}\right)^{\mathcal{A}_{n-1}},\left(S_{n}^{B}\right)^{\mathcal{A}_{n-1}}\right\}$ for some $n \geq 2$. Then by Propositions 2.5, 2.9 and 2.1] we have that $[u, v]^{J} \cong[\Lambda(u), \Lambda(v)] \cong J(\Lambda(v) \backslash \Lambda(u))$, and similarly for $[w, z]^{J}$. Therefore $J(\Lambda(v) \backslash \Lambda(u)) \cong J(\Lambda(z) \backslash \Lambda(w))$ and this, by a wellknown result in the theory of partially ordered sets (see, e.g., [17, Prop. 3.4.2]), implies that $\Lambda(v) \backslash \Lambda(u) \cong \Lambda(z) \backslash \Lambda(w)$, as posets. Assume first that $\Lambda(v) \backslash \Lambda(u)$ is connected. If $|\Lambda(v) \backslash \Lambda(u)| \leq 3$, the result is easy to verify. If $|E(\Lambda(v) \backslash \Lambda(u))| \geq 3$, then by Lemma 4.7 we conclude that $\Lambda(v) \backslash \Lambda(u) \approx \Lambda(z) \backslash \Lambda(w)$ and the result follows from Theorem 4.1. If $|E(\Lambda(v) \backslash \Lambda(u))|=2$, then by Lemma 4.7, and its proof, we conclude that either $\Lambda(v) \backslash \Lambda(u) \approx \Lambda(z) \backslash \Lambda(w)$ or $\Lambda(v) \backslash \Lambda(u) \approx(\Lambda(z) \backslash \Lambda(w))^{\prime}$ and the result follows from Theorem 4.1 and Proposition 3.1. If $\Lambda(v) \backslash \Lambda(u)$ is not connected, then the connected components of $\Lambda(v) \backslash \Lambda(u)$ and $\Lambda(z) \backslash \Lambda(w)$ are pairwise isomorphic as posets, and the result follows from the previous considerations, and the fact that a skew shifted partition is $s$-Dyck if and only if each one of its connected components is $s$-Dyck.

Finally, for the other Hermitian symmetric pairs the result follows immediately from Theorems 4.2 and 4.3 and from computer calculations.

\section{Applications}

In this section we give some consequences of our main results. More precisely, we derive combinatorial closed product formulas for certain alternating sums of ordinary Kazhdan-Lusztig polynomials, and we prove a combinatorial invariance result for certain Kazhdan-Lusztig polynomials studied in [3].

We begin with the following consequence of our main results for the ordinary Kazhdan-Lusztig polynomials.

Corollary 5.1. Let $\left(W, W_{J}\right)$ be a Hermitian symmetric pair, and $u, v \in W^{J}, u \leq v$. Then $\sum_{w \in W_{J}}(-1)^{l(w)} P_{w u, v}(q)$ is either zero or a monic power of $q$.

Proof. This follows immediately from Corollary 4.4 and Proposition 2.3 ,

Note that the exact power of $q$ in Corollary 5.1 is explicitly determined in Theorems 4.1, 4.2 and 4.3, and Theorem 5.1 of [4.

One of the most celebrated conjectures about the Kazhdan-Lusztig polynomials is the so-called "combinatorial invariance conjecture" (see, e.g., [2, p. 161], and the references cited there). This conjecture states that the Kazhdan-Lusztig polynomial $P_{u, v}(q)$ depends only on the isomorphism class of $[u, v]$ as a poset. Using Corollary 4.8 we can prove a (slightly different, and computationally simpler) statement for 
the Kazhdan-Lusztig polynomials studied in [3]. Recall (see, e.g., [2, Prop. 2.4.4]) that given $u \in W$ there exist unique elements $u_{J} \in W_{J}$ and $u^{J} \in W^{J}$ such that $u=u_{J} u^{J}$. Furthermore, $l(u)=l\left(u_{J}\right)+l\left(u^{J}\right)$.

Corollary 5.2. Let $\left(W, W_{J}\right)$ be a Hermitian symmetric pair and $u, v \in W$ be such that $D(u), D(v) \supseteq J$. Then $P_{u, v}(q)$ depends only on

$$
\{x \in[u, v]: D(x) \supseteq J\}
$$

as a poset.

Proof. Let $w, z \in W$ be such that $D(w), D(z) \supseteq J$, and suppose that $\{x \in[w, z]$ : $D(x) \supseteq J\}$ is isomorphic to (25) as a poset. We will show that, then $P_{u, v}(q)=$ $P_{w, z}(q)$. We claim that

$$
\{x \in W: D(x) \supseteq J\} \cong W^{J}
$$

as posets. To see this note first that if $x \in W$, then $D(x) \supseteq J$ if and only if $w_{0}(J) x^{-1} \in W^{J}$, and in this case,

$$
l\left(w_{0}(J) x^{-1}\right)=l\left(x^{-1}\right)-l\left(w_{0}(J)\right)=l(x)-l\left(w_{0}(J)\right) .
$$

In fact, if $D(x) \supseteq J$, then $x s<x$ for all $s \in J$, which implies that $s x^{-1}<x^{-1}$ for all $s \in J$. Writing $x^{-1}=x_{J} x^{J}$ with $x_{J} \in W_{J}$ and $x^{J} \in W^{J}$ we conclude that $s x_{J} x^{J}<$ $x_{J} x^{J}$ for all $s \in J$. But $s x_{J} \in W_{J}$, so $l\left(s x_{J} x^{J}\right)=l\left(s x_{J}\right)+l\left(x^{J}\right)$, which implies that $l\left(s x_{J}\right)<l\left(x_{J}\right)$ for all $s \in J$. Since $x_{J} \in W_{J}$ this implies, by well-known facts (see, e.g., [2, Prop.2.3.1]) that $x_{J}=w_{0}(J)$. Hence $x^{-1}=w_{0}(J) x^{J}$, so $w_{0}(J) x^{-1} \in W^{J}$ and $l\left(x^{-1}\right)=l\left(w_{0}(J)\right)+l\left(x^{J}\right)$. Conversely, if $w_{0}(J) x^{-1} \in W^{J}$, then there exists $y \in W^{J}$ such that $x^{-1}=w_{0}(J) y$. Therefore, for any $s \in J$, we have that $l\left(s x^{-1}\right)=$ $l\left(s w_{0}(J) y\right)=l\left(s w_{0}(J)\right)+l(y)<l\left(w_{0}(J)\right)+l(y)=l\left(x^{-1}\right)$, so $J \subseteq D(x)$. Also, if $x, y \in W$, then $y x^{-1} \in T$ if and only if $w_{0}(J)\left(y^{-1} x\right) w_{0}(J)^{-1} \in T$. This shows, by the definition of Bruhat order and by the fact that both posets in (26) have the chain property $([2$, Thm. 2.5.5]), that if $x, y \in W$ are such that $D(x), D(y) \supseteq J$, then $x \leq y$ if and only if $w_{0}(J) x^{-1} \leq w_{0}(J) y^{-1}$. Therefore the map $x \mapsto w_{0}(J) x^{-1}$ is a poset isomorphism from $\{x \in W: D(x) \supseteq J\}$ to $W^{J}$. Therefore, in particular, $\{x \in[u, v]: D(x) \supseteq J\} \cong\left[w_{0}(J) u^{-1}, w_{0}(J) v^{-1}\right]^{J}$. We therefore conclude from our assumptions that $\left[w_{0}(J) u^{-1}, w_{0}(J) v^{-1}\right]^{J} \cong\left[w_{0}(J) w^{-1}, w_{0}(J) z^{-1}\right]^{J}$. This, by Corollary 4.8. Proposition 2.3. and a well-known property of the Kazhdan-Lusztig polynomials (see, e.g., [2, Chap. 5, Ex. 12]), implies that $P_{u, v}(q)=P_{u^{-1}, v^{-1}}(q)=$ $P_{w_{0}(J) u^{-1}, w_{0}(J) v^{-1}}^{J,-1}(q)=P_{w_{0}(J) w^{-1}, w_{0}(J) z^{-1}}^{J,-1}(q)=P_{w^{-1}, z^{-1}}=P_{w, z}(q)$, as desired.

Note that Corollary 5.2 generalizes, even for Hermitian symmetric pairs of type $A$, Corollary 6.3 of $[4$.

\section{ACKNOWLEDGEMENTS}

Most of the computer calculations in this paper have been carried out using a Maple package for finite Coxeter groups and a Maple package for handling posets developed by John Stembridge. The author would like to thank the referee for a thorough and careful reading of the manuscript. 


\section{REFERENCES}

[1] A. Björner, private communication, March 1992.

[2] A. Björner, F. Brenti, Combinatorics of Coxeter Groups, Graduate Texts in Mathematics, 231, Springer-Verlag, New York, 2005. MR2133266 (2006d:05001)

[3] B. Boe, Kazhdan-Lusztig polynomials for Hermitian symmetric spaces, Trans. Amer. Math. Soc., 309 (1988), 279-294. MR957071 (89i:22024)

[4] F. Brenti, Kazhdan-Lusztig and R-polynomials, Young's lattice, and Dyck partitions, Pacific J. Math., 207 (2002), 257-286. MR1972246(2004e:20008)

[5] F. Brenti, Parabolic Kazhdan-Lusztig R-polynomials for Hermitian symmetric pairs, J. Algebra, 318 (2007), 412-429. MR.2363142

[6] L. Casian, D. Collingwood, The Kazhdan-Lusztig conjecture for generalized Verma modules, Math. Zeit., 195 (1987), 581-600. MR900346 (88i:17008)

[7] V. Deodhar, On some geometric aspects of Bruhat orderings. II. The parabolic analogue of Kazhdan-Lusztig polynomials, J. Algebra, 111 (1987), 483-506. MR916182 (89a:20054)

[8] V. Deodhar, Duality in parabolic setup for questions in Kazhdan-Lusztig theory, J. Algebra, 142 (1991), 201-209. MR.1125213 (92j:20049)

[9] J. Haglund, M. Haiman, N. Loehr, A combinatorial formula for Macdonald polynomials, J. Amer. Math. Soc., 18 (2005), 735-761. MR2138143 (2006g:05223a)

[10] J. Haglund, M. Haiman, N. Loehr, J. Remmel, A. Ulyanov, A combinatorial formula for the character of the diagonal coinvariants, Duke Math. J., 126 (2005), 195-232. MR2115257 (2006f:05186)

[11] J. E. Humphreys, Reflection Groups and Coxeter Groups, Cambridge Studies in Advanced Mathematics, no. 29, Cambridge Univ. Press, Cambridge, 1990. MR1066460 (92h:20002)

[12] M. Kashiwara, T. Tanisaki, Parabolic Kazhdan-Lusztig polynomials and Schubert varieties, J. Algebra, 249 (2002), 306-325. MR1901161(2004a:14049)

[13] D. Kazhdan, G. Lusztig, Representations of Coxeter groups and Hecke algebras, Invent. Math., 53 (1979), 165-184. MR560412 (81j:20066)

[14] B. Leclerc, J.-Y. Thibon, Littlewood-Richardson coefficients and Kazhdan-Lusztig polynomials, Adv. Studies Pure Math., 28 (2000), 155-220. MR.1864481 (2002k:20014)

[15] W. Soergel, Kazhdan-Lusztig polynomials and a combinatoric for tilting modules, Represent. Theory, 1 (1997), 83-114. MR.1444322 (98d:17026)

[16] W. Soergel, Character formulas for tilting modules over Kac-Moody algebras, Represent. Theory, 1 (1997), 115-132. MR1445716 (98f:17016)

[17] R. P. Stanley, Enumerative Combinatorics, vol. 1, Wadsworth and Brooks/Cole, Monterey, CA, 1986.

[18] R. P. Stanley, Enumerative Combinatorics, vol. 2, Cambridge Studies in Advanced Mathematics, no. 62, Cambridge Univ. Press, Cambridge, 1999. MR.1676282 (2000k:05026)

[19] M. Varagnolo, E. Vasserot, On the decomposition matrices of the quantized Schur algebra, Duke Math. J., 100 (1999), 267-297. MR.1722955(2001c:17029)

Dipartimento di Matematica, Università di Roma “Tor Vergata”, Via della Ricerca Scientifica, 1, 00133 Roma, Italy

E-mail address: brenti@mat.uniroma2.it 